\title{
Differential Coding Strategies in Glutamatergic and GABAergic Neurons in the Medial Cerebellar Nucleus
}

\author{
Orçun Orkan Özcan, ${ }^{1}$ Xiaolu Wang, ${ }^{2}$ Francesca Binda, ${ }^{1}$ Kevin Dorgans, ${ }^{1}$ Chris I. De Zeeuw, ${ }^{2,3}$ Zhenyu Gao, ${ }^{2}$ \\ DAd Aertsen, ${ }^{4,5}{ }^{\circledR}$ Arvind Kumar, ${ }^{5,6}$ and ${ }^{\circ}$ Philippe Isope ${ }^{1}$ \\ ${ }^{1}$ Centre National de la Recherche Scientifique, Université de Strasbourg, Institut des Neurosciences Cellulaires et Intégratives, F-67000 Strasbourg, France, \\ ${ }^{2}$ Department of Neuroscience, Erasmus MC, 1105 BA Rotterdam, The Netherlands, ${ }^{3}$ Netherlands Institute for Neuroscience, Amsterdam, The Netherlands, \\ ${ }^{4}$ Faculty of Biology, University of Freiburg, Freiburg 79104, Germany, ${ }^{5}$ Bernstein Center Freiburg, University of Freiburg, Freiburg 79104, Germany, and \\ ${ }^{6}$ Computational Science and Technology, School of Electrical Engineering and Computer Science, KTH Royal Institute of Technology, Stockholm SE-100 44, \\ Sweden
}

The cerebellum drives motor coordination and sequencing of actions at the millisecond timescale through adaptive control of cerebellar nuclear output. Cerebellar nuclei integrate high-frequency information from both the cerebellar cortex and the two main excitatory inputs of the cerebellum: the mossy fibers and the climbing fiber collaterals. However, how nuclear cells process rate and timing of inputs carried by these inputs is still debated. Here, we investigate the influence of the cerebellar cortical output, the Purkinje cells, on identified cerebellar nuclei neurons in vivo in male mice. Using transgenic mice expressing Channelrhodopsin2 specifically in Purkinje cells and tetrode recordings in the medial nucleus, we identified two main groups of neurons based on the waveform of their action potentials. These two groups of neurons coincide with glutamatergic and GABAergic neurons identified by optotagging after Chrimson expression in VGLUT2-cre and GAD-cre mice, respectively. The glutamatergic-like neurons fire at high rate and respond to both rate and timing of Purkinje cell population inputs, whereas GABAergic-like neurons only respond to the mean population firing rate of Purkinje cells at high frequencies. Moreover, synchronous activation of Purkinje cells can entrain the glutamatergic-like, but not the GABAergic-like, cells over a wide range of frequencies. Our results suggest that the downstream effect of synchronous and rhythmic Purkinje cell discharges depends on the type of cerebellar nuclei neurons targeted.

Key words: cerebellar nuclei; cerebellum; electrophysiology in vivo; Purkinje cells; temporal coding

Significance Statement

Motor coordination and skilled movements are driven by the permanent discharge of neurons from the cerebellar nuclei that communicate cerebellar computation to other brain areas. Here, we set out to study how specific subtypes of cerebellar nuclear neurons of the medial nucleus are controlled by Purkinje cells, the sole output of the cerebellar cortex. We could isolate different subtypes of nuclear cell that differentially encode Purkinje cell inhibition. Purkinje cell stimulation entrains glutamatergic projection cells at their firing frequency, whereas GABAergic neurons are only inhibited. These differential coding strategies may favor temporal precision of cerebellar excitatory outputs associated with specific features of movement control while setting the global level of cerebellar activity through inhibition via rate coding mechanisms

\section{Introduction}

The cerebellum plays a major role in the control, timing, and learning of skilled movements (Ito, 1984; Thach et al., 1992;

Received April 10, 2019; revised 0ct. 8, 2019; accepted 0ct. 30, 2019.

Author contributions: 0.0.0., X.W., F.B., and K.D. performed research; 0.0.0., X.W., F.B., K.D., Z.G., and P.I. analyzed data; 0.0 .0. and P.I. wrote the first draft of the paper; 0.0.0., X.W., F.B., C.I.D.Z., Z.G., A.A., A.K., and P.I. edited the paper; 0.0.0. and P.I. wrote the paper; C.I.D.Z., A.A., and A.K. contributed unpublished reagents/analytic tools; Z.G., A.K., and P.I. designed research.

This work was supported by Centre National pour la Recherche Scientifique, Université de Strasbourg, Agence Nationale pour la Recherche Grant ANR-15-CE37-0001-01 CeMod, and Fondation pour la Recherche Médicale DEQ20140329514 to P.I. 0.0.0. was supported by the NeuroTime Erasmus Mundus Joint Doctorate Neuroscience
Spencer et al., 2003; Bastian, 2006) via a constant dialog with the cerebral cortex (Allen and Tsukahara, 1974; Schmahmann and Pandya, 1997; Kelly and Strick, 2003; Glickstein et al., 2011;
PhD program (funded by the European Commission). C.I.D.Z. was supported by European research council-advanced (ERC-adv), European research council proof of concept (ERC-POC), Dutch research council (NWO-ALW), The Netherlands Organisation for Health Research and Development (ZonMw), Koninklijke Nederlandse Akademie van Wetenschappen (KNAW). We thank Clément Léna for technical and conceptual discussions; Matilde Cordero-Erausquin Frédéric Doussau, and Didier De Saint Jan for critical readings; and Sophie Reibel-Foisset and the Chronobiotron (UMS 3415, (entre National de la Recherche Scientifique) for mice handling.

The authors declare no competing financial interests.

F. Binda's present address: Department for Biomedical Research, Murtenstrasse 40, 3008 Bern CH. 
Proville et al., 2014). The cerebellar nuclei generate the final output of the cerebellum by integrating inputs from inhibitory Purkinje cells (PCs) with those of the mossy and climbing fiber collaterals, the two main inputs of the cerebellum (Gauck and Jaeger, 2000; Rowland and Jaeger, 2005; Bengtsson et al., 2011; Steuber and Jaeger, 2013; Bengtsson and Jörntell, 2014) (see Fig. $1 A)$. CN neurons seem to sum input rate from PCs and encode some part of the movement as a rate code (Thach, 1968; Armstrong and Edgley, 1984; Brooks and Thach, 2011; Abbasi et al., 2017) or follow the temporal pattern of synchronous PC discharges (Heck et al., 2007; De Zeeuw et al., 2008, 2011; Person and Raman, 2011, 2012; Sarnaik and Raman, 2018) or pauses (Shin and De Schutter, 2006; Steuber et al., 2007; Hong et al., 2016) as observed in delay eye-blink conditioning (McCormick and Thompson, 1984; Yeo et al., 1997; Rasmussen et al., 2015; Ten Brinke et al., 2015, 2017). Indeed, disrupting the temporal pattern of PCs or CN neurons discharges strongly affects motor coordination and leads to ataxia (Hoebeek et al., 2005; Walter and Khodakhah, 2006; Walter et al., 2006; Chen et al., 2010; White et al., 2014; White and Sillitoe, 2017). However, whether rate-based or temporal coding strategies are associated with a specific task or with specific physiological properties of CN neuron subtypes has not been established yet as the distinction between cell types in the $\mathrm{CN}$ remains difficult.

$\mathrm{CN}$ neurons have been subdivided into six subgroups, of which five are present in the medial nucleus: glutamatergic, GABAergic nucleo-olivary and glycinergic projection neurons, and local GABAergic and/or glycinergic cells (Czubayko et al., 2001; Uusisaari et al., 2007; Alviña et al., 2008; Uusisaari and Knöpfel, 2008, 2011; Bagnall et al., 2009; Tadayonnejad et al., 2010; Zheng and Raman, 2010; Blenkinsop and Lang, 2011; Bengtsson and Jörntell, 2014; Husson et al., 2014; Ankri et al., 2015; Najac and Raman, 2015; Canto et al., 2016; YardenRabinowitz and Yarom, 2017). Specific intrinsic biophysical properties (e.g., spike width, afterhyperpolarization shape and duration, firing rate) for at least 4 different $\mathrm{CN}$ neurons subtypes have been described using intracellular recordings (Uusisaari et al., 2007; Uusisaari and Knöpfel, 2008, 2011; Bagnall et al., 2009; Ankri et al., 2015; Najac and Raman, 2015; Canto et al., 2016; Yarden-Rabinowitz and Yarom, 2017). However, in recent in vivo studies in awake animals (Bengtsson et al., 2011; Bengtsson and Jörntell, 2014; Brown and Raman, 2018; Sarnaik and Raman, 2018), only glutamatergic projection neurons could be reliably recorded. This illustrates the difficulties in discrimination of neuronal subtypes using extracellular recordings as opposed to the cerebral cortex in which GABAergic versus glutamatergic neurons are reliably distinguished by their spike waveforms (see, e.g., Barthó et al., 2004). We therefore set out to identify simple criteria allowing us to discriminate between excitatory (glutamatergic [GLUT]) projection neurons and inhibitory (GABAergic [GABA]) interneurons, and to characterize how these cell types respond to $\mathrm{PC}$ inputs.

We recorded $\mathrm{CN}$ neurons in the medial nucleus of anesthetized and awake animals expressing channelrhodopsin2 (ChR2) in PCs (Chaumont et al., 2013) or Chrimson either in GLUT or in GABA cells in $\mathrm{CN}$, respectively. We found that GLUT versus GABA neurons could be segregated by their average spike wave-

K. Dorgans's present address: Okinawa Institute of Science and Technology Graduate University, Okinawa, Japan 904-0495.

Correspondence should be addressed to Philippe Isope at philippe.isope@inci-cnrs.unistra.fr.

https://doi.org/10.1523/JNEUROSCI.0806-19.2019

Copyright $\odot 2020$ the authors forms and have significant differences in their firing rates. While both groups were inhibited by PC inputs, only GLUT-like neurons closely followed the synchronous spiking of PCs. That is, only GLUT-like neurons combine PC inhibition with spike discharge, which was time-locked to PC population input, whereas GABA-like cells are not sensitive to the timing of PC discharges at high frequencies. Together, our findings suggest that several coding strategies are multiplexed in $\mathrm{CN}$ neurons, depending on the cell-type targeted, allowing for a simultaneous control of firing rate and temporal discharge.

\section{Materials and Methods}

Animals. All procedures were in accordance with national and European community guidelines (2010/63/EU). Animal experiments were approved in advance by the local French Ethics Committee of the University of Strasbourg (C.R.E.M.E.A.S.; CEEA35; agreement number/ reference protocol: A67-2018-38). Mice were bred and housed in a $12 \mathrm{~h}$ light/dark cycle with free access to food and water. We used 13 L7ChR2(H134R)-YFP CD1 male mice (Chaumont et al., 2013) or adult C57BL/6 GAD2-IRES-Cre $(n=6$; stock \#010802) and VGLUT2IRES-Cre $(n=4$; stock \#016963) mice (The Jackson Laboratory; RRID:SCR_004633).

Viral injection. PC connections were traced from lobules IV and V of the cerebellar vermis to the medial CN using AAV 2/1-GFP (PENN Vector Core; RRID:SCR_010038) or DiI (lipophilic cationic indocarbocyanine dye; Thermo Fisher Scientific). Mice were anesthetized by an intraperitoneal injection of a mixture of ketamine (100 mg/kg), medetomidine $(1 \mathrm{mg} / \mathrm{kg})$, and acepromazine $(3 \mathrm{mg} / \mathrm{kg})$ and positioned on a stereotaxic frame. The tracers were loaded into a graduated pipette equipped with a piston for manual injections (Wiretrol II, Drummond Scientific). By applying gentle pressure, a final volume of $0.5 \mu \mathrm{l}$ was delivered into the tissue at an approximate speed of $250 \mathrm{nl} / \mathrm{min}$. The pipette was left in place for at least $10 \mathrm{~min}$ after injection for diffusion. After 2 weeks (AAV2/1-GFP) or 2-3 d (DiI), injected mice were killed by transcardiac perfusion of PFA $4 \%$, and cerebellar slices $(50 \mu \mathrm{m})$ were prepared for subsequent analysis. Serial confocal acquisitions were performed (Leica Microsystems, SP5) onto sequential sections of cerebellar slices. Some Purkinje cells were individually traced using the ImageJ (National Institutes of Health) plugin "Simple neurite tracer" (RRID: SCR_016566) and stereotaxic coordinates of the connected regions in the cerebellar cortex and the medial $\mathrm{CN}$ were obtained.

For GAD2-cre and VGLUT2-cre mice intracranial injection, anesthesia was induced with $4 \%$ isoflurane inhalation and maintained with $2.5 \%$ during the operation. Animals were fixed on a stereotaxic frame. Rectal temperature was monitored and kept at $36^{\circ} \mathrm{C}-37.5^{\circ} \mathrm{C}$. A small craniotomy ( $1.5 \mathrm{~mm}$ diameter) was applied at the coordinates of fastigial nucleus (AP $2.6 \mathrm{~mm}$, ML $0.8 \mathrm{~mm}$, DL $2.4 \mathrm{~mm}$ from lambda), except for 1 cell recorded in the interposed nucleus (AP $2.4 \mathrm{~mm}$, ML $2.1 \mathrm{~mm}$, DL $2.3 \mathrm{~mm}$ from lambda). A capillary ( $80 \mu \mathrm{m}$ tip diameter) containing AAV9-hSyn-FLEX-ChrimsonR-tdTomato (UNC Vector Core; RRID: SCR_002448) was inserted vertically in the craniotomy, and a volume of $60 \mathrm{nl}$ of virus was slowly injected in CN. The capillary was left at least 10 min after injection for diffusion. Postsurgically buprenorpine $(4 \mathrm{mg} / \mathrm{kg}$, i.p.) was injected. Animals were allowed to recover at least 4 weeks before experiments.

Optogenetic stimulation in anesthetized animals (L7-ChR2(H134R)YFP). Optical stimulation was performed using a multimode optical fiber (100 $\mu \mathrm{m}$ diameter) positioned with a manual micromanipulator above the surface of the cerebellar cortex and mounted with a blue LED at 460 nm wavelength (UHP-T-LED 460, Prizmatix). Light intensity was set $<25 \mathrm{~mW} / \mathrm{mm}^{2}$ to avoid depolarization block in PCs (Chaumont et al., 2013). Each episode (10 s duration including $7 \mathrm{~s}$ of stimulation) consisted of a train of pulses ( $5 \mathrm{~ms}$ pulse duration) delivered either regularly at 40 , 65 , and $115 \mathrm{~Hz}$ frequencies or irregularly at $40 \mathrm{~Hz}$ (following a Poisson distribution). For each stimulation frequency, at least 10 trials were performed for every recorded cell. Illumination protocols were executed using a single board computer (Raspberry Pi). 
Optogenetic stimulation in awake animals (GAD2-IRES-Cre and VGLUT2-IRES-Cre). For GABA and GLUT neuron optotagging, animals were surgically implanted with $2 \mathrm{~mm}$ length optical fiber $(\varnothing 105 \mu \mathrm{m}$, ThorLabs) in the cerebellar cortex, above the CN. A $595 \mathrm{~nm}$ wavelength LED driven by a DC2100 controller (M595F2, ThorLabs) was connected to the optical fiber. Each episode ( $2 \mathrm{~s}$ duration) of stimulation consisted of a train of pulses ( $5 \mathrm{~ms}$ pulse duration) at $100 \mathrm{~Hz}$ for $125 \mathrm{~ms}$ repeated at least 10 times every 5-10 s. We only included neurons that responded to optogenetic stimulation with a short latency $(<20 \mathrm{~ms})$ (Klapoetke et al., 2014; Mardinly et al., 2018). The latency to optogenetic stimulation was defined as the first epoch that exceeded 3 times the SD of the baseline firing rate. Assessment of firing rate change was based on peristimulus time histogram (constructed with at least 10 trials).

Tetrode recordings in anesthetized L7-ChR2(H134R)-YFP mice. Mice were deeply anesthetized under urethane $(1.9 \mathrm{~g} / \mathrm{kg}$ i.p. $)$. Tracheotomy was performed to facilitate breathing. Mice were placed in a stereotaxic frame (Narashige). Body temperature was maintained using a heating blanket (CWE, TC-1000) and monitored by a rectal probe. Craniotomy was then performed to uncover the dorsal vermis and paravermis of the cerebellar cortex. After incision of the dura, the tissue was constantly moisturized with a saline solution $(\mathrm{NaCl} 9 \mathrm{~g} / \mathrm{L})$. PCs and $\mathrm{CN}$ cells were recorded using custom-designed silicon probes $(2 \times 16$ channels, Atlas Neuroengineering) motorized with a manipulator (Luigs and Neuman, SM5). Each probe (tetrode) was composed of 4 shanks ( $150 \mu \mathrm{m}$ between shanks) bearing 4 circular iridium oxide recording sites ( $15 \mu \mathrm{m}$ diameter) separated by $20 \mu \mathrm{m}$ in a diamond shape. One probe was lowered into lobules IV and V in the vermis to record PCs. The second one was lowered through lobule VI in the paravermis to record $\mathrm{CN}$ neurons. Both probes were positioned using motorized micromanipulators (Luigs and Neumann). Data acquisition was performed using a portable 32 channel acquisition amplifier (ME32-FAI- $\mu$ PA, MultiChannel Systems) at 20 $\mathrm{kHz}$ sampling rate and $5 \mathrm{kHz}$ low pass filtering. The probes were painted with DiI for post hoc reconstruction of the recording sites.

Juxtacellular and multichannel recordings in awake GAD2-IRES-Cre and VGLUT2-IRES-Cre mice. A vertical skin cut was applied to expose skull. The skull was pretreated with Optibond All-in-one (Kerr), and a $5.5 \times 4.0 \mathrm{~mm}$ custom-made pedestal was attached to the skull with Charisma (Heraeus Kulzer). Mice were allowed to recover at least 2 weeks after surgery and before recordings. Before electrophysiology experiments, a small craniotomy $(\Phi=2.0 \mathrm{~mm})$ was performed on the skull over recording targets. Mice were head fixed but allowed to move on a cylinder treadmill. GABA and GLUT cells were recorded with glass capillary ( $\varnothing=2 \mathrm{~mm}$, tip $=2 \mu \mathrm{m}$, filled with $2 \mathrm{~m}$ saline; $n=15$ cells) electrodes as previously described (Ten Brinke et al., 2017) or with a 64-channel silicon probe (ASSY 77H-H2, Cambridge NeuroTech; $n=2$ cells). The electrode was motorized with a manipulator (Luigs and Neuman, SM7) and penetrated the cerebellar nuclei with an angle of $35^{\circ}$ from vertical axis. For glass capillary recordings, neuronal electrophysiological signals were amplified (Axon Instruments CNS, MultiClamp 700B, AutoMate Scientific US) and digitized at $20 \mathrm{kHz}$ with an Axon Instruments Digidata acquisition system. For silicon probe recordings, signals were amplified and digitized at $20 \mathrm{kHz}$ with an Intan evaluation system (RHD2000, Intan Technology).

Data analysis. Spike sorting was performed using the open source software OpenElectrophy (RRID:SCR_000819) (Garcia and FourcaudTrocmé, 2009). Before spike sorting, stimulation artifacts were removed as described by Rowland and Jaeger (2008). Raw data for each tetrode were high-pass filtered $(200 \mathrm{~Hz})$ using a Butterworth filter. A manual threshold was determined by visually inspecting the signal, after which peaks crossing the threshold were detected. Spike waveforms were collected ( $\pm 1 \mathrm{~ms}$ around the peaks) and classified after normalization at the positive peak time and amplitude using principal component analysis (PCA) using the scikit-learn package (Pedregosa et al., 2011). Spike waveforms were sorted into independent units using Gaussian Mixture Models based on the expectation maximization algorithm (Python scikit-learn package). Individual units were always confirmed by visual inspection, and we took a conservative approach and kept only wellseparated clusters.
Average spike waveforms from all recorded units (cells) were classified using PCA followed by Hierarchical Clustering on Principal Components (Lê et al., 2008) in R statistical software (R Core Team, 2015) based on parameters extracted from the average spike waveforms (see Fig. 2D). Other analyses were performed using custom routines written in Python. Instantaneous firing rate (IFR) was calculated as the inverse of the interval between consecutive spikes.

Immunofluorescence. Animals were killed and perfused transcardially with $50 \mathrm{ml}$ saline, followed by $100 \mathrm{ml}$ of $4 \%$ PFA/0.01 м PBS, pH 7.4. Then, the brain was removed immediately and postfixed for $2 \mathrm{~h}$ at room temperature. The fixed brain dehydrated in $10 \%$ sucrose overnight at $4^{\circ} \mathrm{C}$, then was embedded in $10 \%$ sucrose $/ 12 \%$ gelatin, and further dehydrated in $30 \%$ sucrose solution. Next, transverse sections $(40 \mu \mathrm{m})$ were cut with a microtome (Leica Microsystems, CM1950). Sections were incubated for $24 \mathrm{~h}$ with rabbit-anti-Gad65/67* (1:1000, Millipore AB1511; RRID:AB_90715). Next, sections were incubated for $12 \mathrm{~h}$ in 5\% normal donkey serum- $0.4 \%$ Triton X-100-0.01 M PBS with A488conjugated donkey anti-rabbit IgG (1:200, Jackson ImmunoResearch Laboratories, lot \#711-545-152). All incubations were performed at $4^{\circ} \mathrm{C}$ with continuous gentle agitation and followed by $3 \times 10$ min washes in PBS. Eventually, sections were stained with DAPI and mounted with VECTASHIELD Mounting Medium (Vector Laboratories, lot \#H-1000).

Statistical analyses. Spike time-locking was assessed by detecting a nonuniform distribution of spikes in the interval between successive stimulations during periodic pulse trains using the Rayleigh test (using Scipy Python package) (Millman and Aivazis, 2011; see also Najac and Raman, 2015). Kolmogorov-Smirnov tests on IFR distributions (see Fig. 6) were performed with a bin of $5 \mathrm{~Hz}$. Statistical tests between groups are described in Results.

\section{Results}

\section{Optogenetic excitation of PCs in lobule IV-V inhibit CN neurons in the medial nuclei}

Using the L7-ChR2(H134R)-YFP strain of mice in which ChR2 was specifically expressed in PCs, we recorded the activity of $\mathrm{CN}$ neurons in anesthetized mice in the medial nucleus while optically stimulating PCs in lobules IV and V. To facilitate probe positioning in the medial $\mathrm{CN}$ in $\mathrm{CD} 1$ mice for which a complete stereotaxic atlas is not available, $\mathrm{PC}$ tract was traced using AAV1/ 2-GFP or DiI injections ( $N=2$ animals). We determined that, in CD1 mice, the caudodorsal medial nucleus can be targeted by inserting the silicon probe $600 \mu \mathrm{m}$ posterior to lobules IV and V at $2.8-3.3 \mathrm{~mm}$ depth (Fig. $1 B$, bottom; $n=25$ recordings sites, $N=13$ animals). Electrode positioning and blue light power were physiologically assessed by simultaneously recording multiunit activity in PCs and in CN neurons (Fig. 1C).

Light-induced (5 ms pulses at 40,65, and $115 \mathrm{~Hz}$ ) PC discharge was assessed in a group of PCs $(n=10)$ using multiunit recordings in the PC layer in the illumination area (Fig. $1 D$, E; see Materials and Methods). No depolarization block was observed, and the averaged firing rate increased compared with baseline (mean \pm SD, baseline: $46.5 \pm 33.8 \mathrm{~Hz} ; 40 \mathrm{~Hz}$ : $65.6 \pm 40.1 \mathrm{~Hz} ; 65$ Hz: $65.2 \pm 36.9 \mathrm{~Hz} ; 115 \mathrm{~Hz}: 57.1 \pm 34.3 \mathrm{~Hz}$ ). Although individual PCs did not follow the stimulation at the highest frequency, by building interspike interval (ISI) histograms (Fig. 1D) and the histogram of the spike times between 2 pulses of light, we observed that 8 of 10 PCs were time-locked (i.e., synchronized) to PC illuminations at high frequency (Fig. $1 E$ ). Therefore, considering that illumination activates tens of PCs (see Chaumont et al., 2013), we estimate that the PC population can follow illumination at all frequencies tested. We then used tetrode recordings and isolated 39 individual cells based on their averaged spike waveforms in the caudodorsal medial nucleus $(N=13$ animals; Fig. $2 A-C$ ). 
A

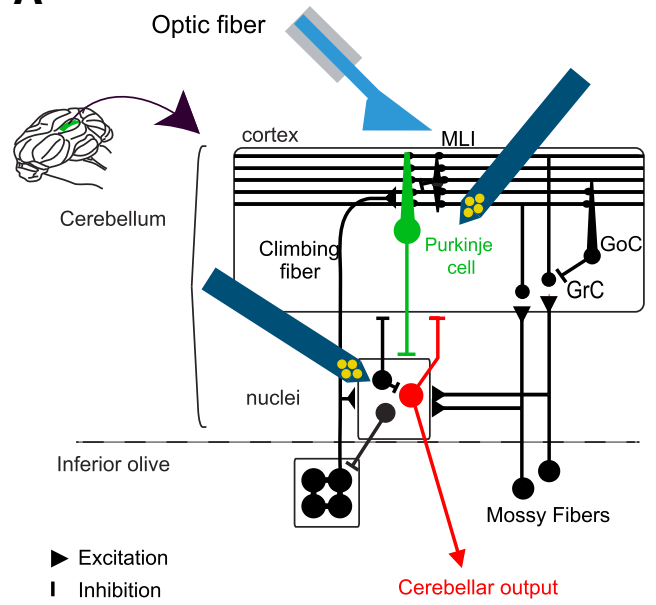

B

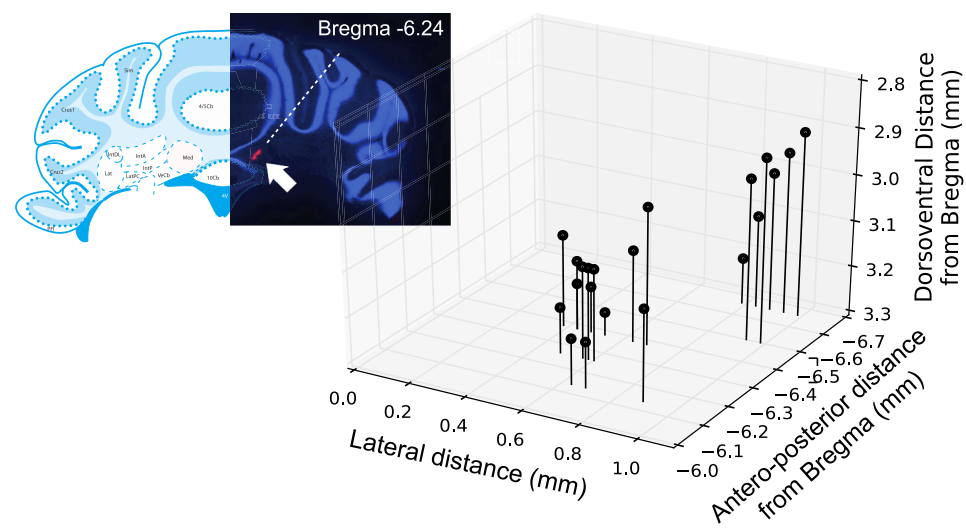

C

Purkinje cell

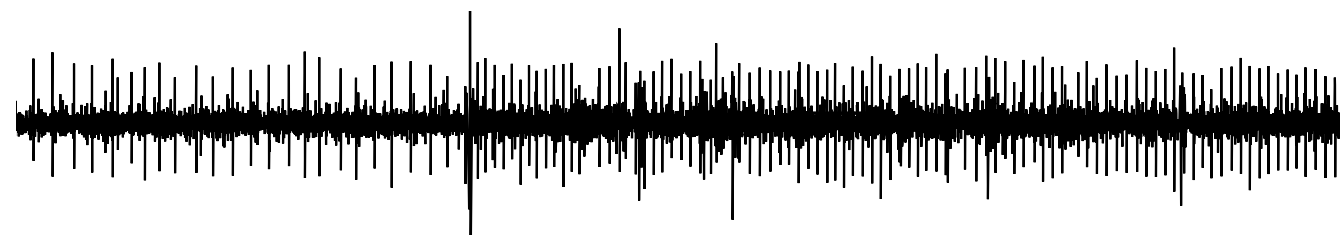

$\mathrm{CN}$ cell
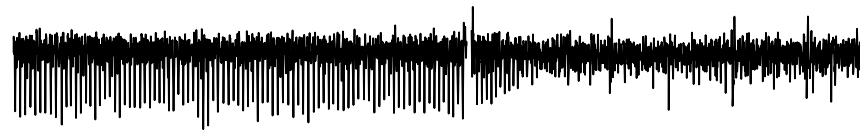

D

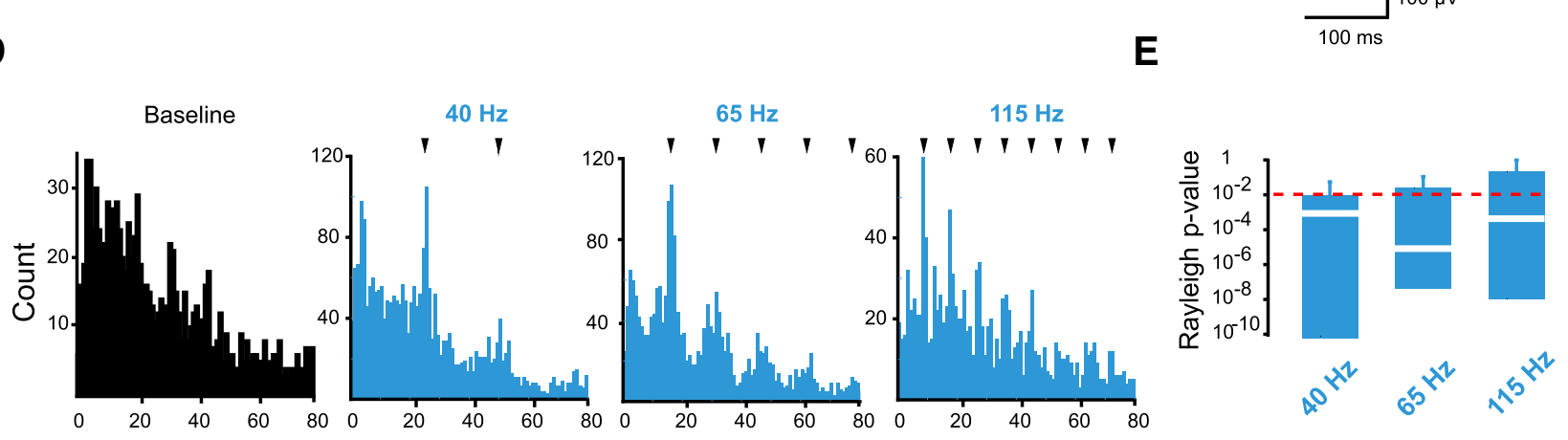

ISI (ms)

Figure 1. Optogenetic stimulation of PCs inhibits CN neurons in the medial nucleus. A, Diagram of the olivo-cerebellar network and experimental protocol for tetrode recordings. Recordings (dark blue) are simultaneously performed in the PC layer and the medial nucleus while an optic fiber (light blue) is positioned above the cerebellar cortex. MLI, Molecular interneurons; GoC, Golgi cells; GrC, granule cells. $\boldsymbol{B}$, Tetrode position was reconstructed after the experiment. Left, Tetrode track monitored by Dil fluorescence (red) in a cerebellar section stained with DAPI. Right, 3D scatter plot of the coordinates of the recording sites. C, Example of multiunit recordings in the $\mathrm{PC}$ layer and medial CN. D, Example of interstimulus spike histogram (ISI) of $\mathrm{PC}$ discharge under light stimulation at 40 , 65 , and $115 \mathrm{~Hz}$. E, Boxplot of $p$ values for each cell from Rayleigh test calculated from the histograms of the spike latency from stimulation onset at 40,65 , and $115 \mathrm{~Hz}$. $p<0.01$ indicates nonuniform distribution (dotted red line). White line indicates median. Box limit indicates first (Q1) and third (Q3) quartile. Whiskers represent $1.5 \times(03-01)$ from the edge of the box.

\section{Discriminating GLUT versus GABA neurons}

To classify CN cells, a PCA was performed on parameters of the averaged spike waveforms (Fig. 2D,E), followed by hierarchical clustering based on the first two principal components (see Materials and Methods). The hierarchical clustering identified two groups of averaged waveforms (Group 1: 22 cells; Group 2: 17 cells; $N=13$ animals). The two groups could be discriminated by the decay phase of the spike waveform (e.g., the negative halfwidth; Fig. 2D, F; Table 1), with Group 1 having a faster action potential than Group 2 neurons (action potential width Group 1:
$0.47 \pm 0.08 \mathrm{~ms}$ vs Group 2: $0.65 \pm 0.11 \mathrm{~ms})$. Next, we analyzed the spike statistics of individual cells belonging to these two groups separately and found that Group 1 and Group 2 cells had significantly different firing rates (Fig. 2G; mean Group 1:63.9 \pm $23.3 \mathrm{~Hz}$; mean Group 2: $23.2 \pm 19.4 \mathrm{~Hz}$; Wilcoxon rank test, $p=$ $\left.1.4 \times 10^{-7}\right)$, whereas the spike train regularity was similar for the two groups (mean CV Group 1: $0.58 \pm 0.42$ vs Group 2: $0.59 \pm$ 0.35; Wilcoxon rank test, $p=0.37$; mean CV2 Group 1: $0.43 \pm$ 0.22 vs Group 2: $0.42 \pm 0.24$; Wilcoxon rank test, $p=0.36$ ). Only 3 pairs of cells belonging to different groups were recorded on the 


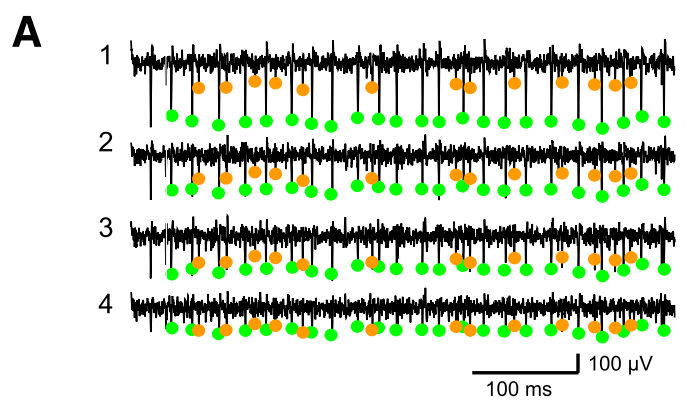

D

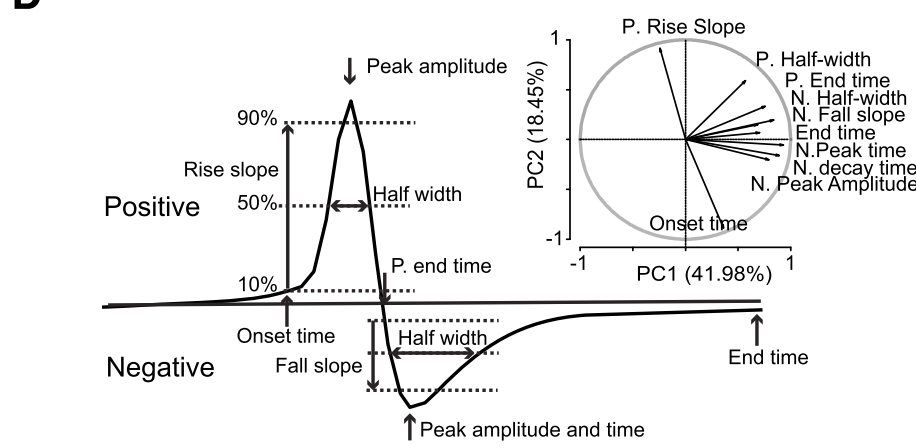

$\mathbf{F}$

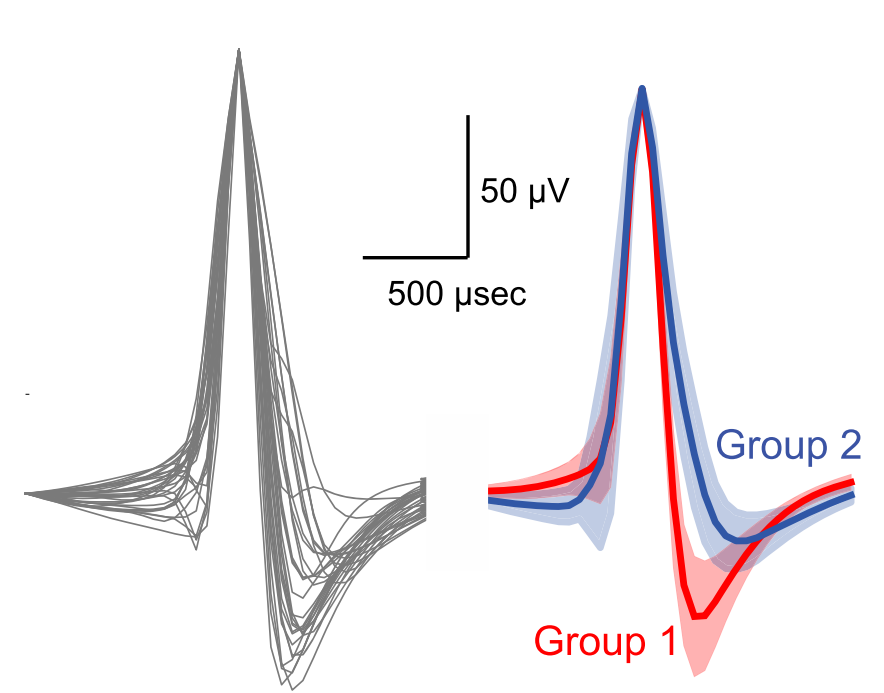

B

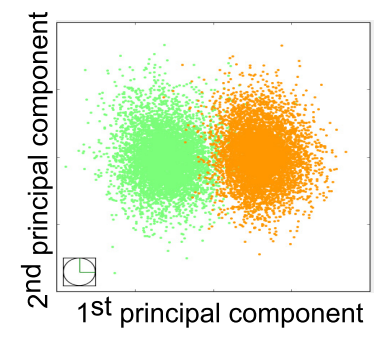

C

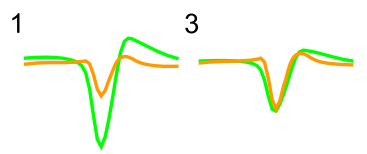

2

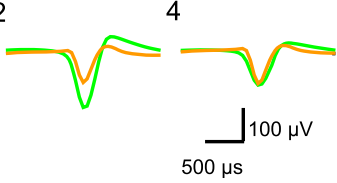

E

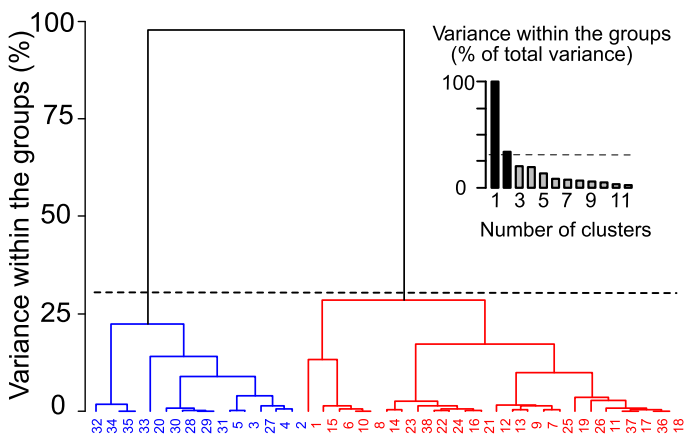

G

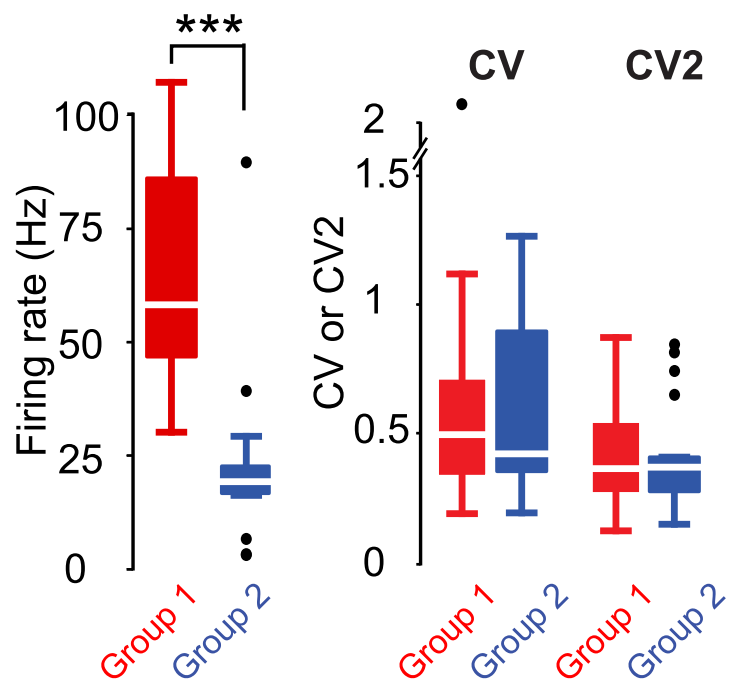

Figure 2. Tetrode recordings identify two distinct groups of $\mathrm{CN}$ neurons. $A$, Example of unit isolation: raw signal on the 4 recording sites of a single tetrode. Orange and blue dots represent spikes from the 2 units isolated by the PCA (Materials and Methods). B, Projection of the spikes from the 2 units against the first 2 principal components. C, Averaged spike waveforms of the 2 units on the 4 recording sites. $\boldsymbol{D}$, Averaged waveforms for all the recorded units were classified using PCA followed by a hierarchical clustering on principal components (HCPC). Description of the measured parameters for the PCA and projection on the first two principal components (top right). Onset time, Time point where the spike waveform reached $5 \%$ of its positive peak; P. Rise slope, positive $10 \%-90 \%$ rise time; P. Half-width, spike duration at $50 \%$ of the positive peak; N. Peak amplitude, negative peak; Negative Peak time, time of negative peak; N. fall slope, $10 \%-90 \%$ fall time; N. Half-width, spike duration at $50 \%$ of the negative peak; N. Decay time, spike duration from negative peak to $10 \%$ of the negative peak; End time, time point at $5 \%$ of its negative peak. $\boldsymbol{E}$, Ascending hierarchical tree highlighting 2 groups of action potential waveforms. $\boldsymbol{F}$, Superimposed averaged spike waveforms of all units normalized to the maximum amplitude (left); mean of the averaged spike waveforms for the 2 groups (Group 1, red, $n=22$; Group 2, blue, $n=17$ ) (right) identified. Shaded area represents SD. G, Boxplot of firing rates, CV and CV2 for the 2 groups. Wilcoxon rank test, Firing rate: ${ }^{* *} p=1.4 \times 10^{-7} ; \mathrm{CV}: p=0.37 ; \mathrm{CV} 2: p=0.36$.

same tetrodes, and no pairwise spike correlation was observed between the cells of these pairs.

In a separate series of experiments in awake animals, we assessed whether the GLUT versus GABA identity of the cells could account for this segregation in two groups. Specific expression of Chrimson in GLUT or GABA CN neurons was obtained by injecting AAV9-hSyn-FLEX-ChrimsonR-tdTomato virus in the CN of VGLUT2-cre and GAD2-cre mice, respectively (Fig. 3). CN neurons were extracellularly recorded and opto-tagged in awake animals (see Materials and Methods). Opto-tagging (GLUT vs
GABA) was confirmed by an instantaneous increase in firing rate under blue light illumination (mean \pm SD delay after light onset GLUT: $6.8 \pm 5.3 \mathrm{~ms}, n=6$; GABA: $5 \pm 4.2 \mathrm{~ms}, n=11$; Fig. $3 A$ ). We determined the averaged spike waveforms of the GLUT and GABA neurons and measured the 5 most relevant parameters that were identified by PCA in the dataset recorded earlier using tetrodes (Fig. 3C; Table 1; $n=15$ ). GLUT and GABA neurons were discriminated by the same set of action potential waveform parameters that earlier discriminated the neurons recorded in anesthetized animals using tetrodes into two groups (compare 
Table 1. Averaged spike waveform parameters for GLUT and GABA neurons obtained by optotagging in VGLUT2-cre/GAD-cre mice or GLUT-like and GABA-like recorded in L7-ChR2-(H134R)-YFP mice using tetrodes ${ }^{a}$

\begin{tabular}{|c|c|c|c|c|c|c|}
\hline & \multicolumn{3}{|c|}{ VGLUT2-cre/GAD-cre } & \multicolumn{3}{|l|}{ L7-ChR2-YFP } \\
\hline & GLUT & GABA & $p$ value (WMW) & GLUT-like & GABA-like & $p$ value (WMW) \\
\hline Positive decay time (ms) & $0.10 \pm 0.03$ & $0.17 \pm 0.06$ & 0.0156 & $0.14 \pm 0.03$ & $0.26 \pm 0.06$ & 0.000001 \\
\hline Negative peak time (ms) & $1.29 \pm 0.04$ & $1.42 \pm 0.09$ & 0.0103 & $1.33 \pm 0.05$ & $1.54 \pm 0.07$ & 0.00000017 \\
\hline Positive end time (ms) & $1.16 \pm 0.03$ & $1.24 \pm 0.07$ & 0.0157 & $1.21 \pm 0.04$ & $1.37 \pm 0.07$ & 0.00000027 \\
\hline Negative fall slope (ms) & $0.07 \pm 0.00$ & $0.11 \pm 0.02$ & 0.0037 & $0.07 \pm 0.01$ & $0.10 \pm 0.02$ & 0.00000032 \\
\hline Negative half-width (ms) & $0.24 \pm 0.06$ & $0.37 \pm 0.10$ & 0.0012 & $0.30 \pm 0.07$ & $0.44 \pm 0.05$ & 0.0000026 \\
\hline
\end{tabular}

${ }^{a}$ WMW, Wilcoxon-Mann-Whitney rank test between $\mathrm{GLUT}_{\text {(like) }}$ and $\mathrm{GABA}_{\text {(like) }}$ groups. Positive decay time, time from the positive peak to $10 \%$ of the positive waveform.

A

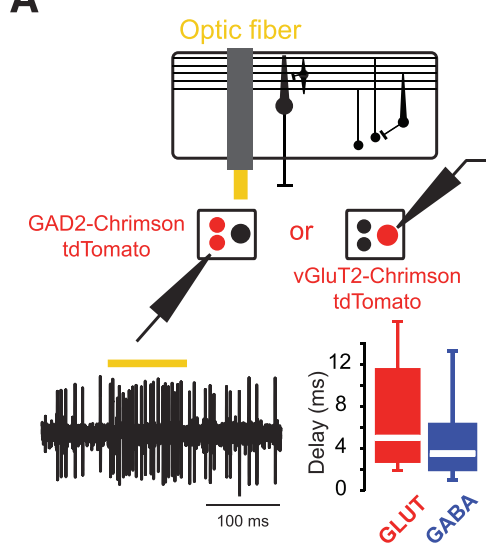

C

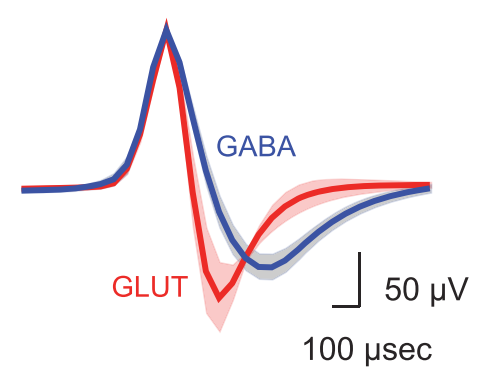

B
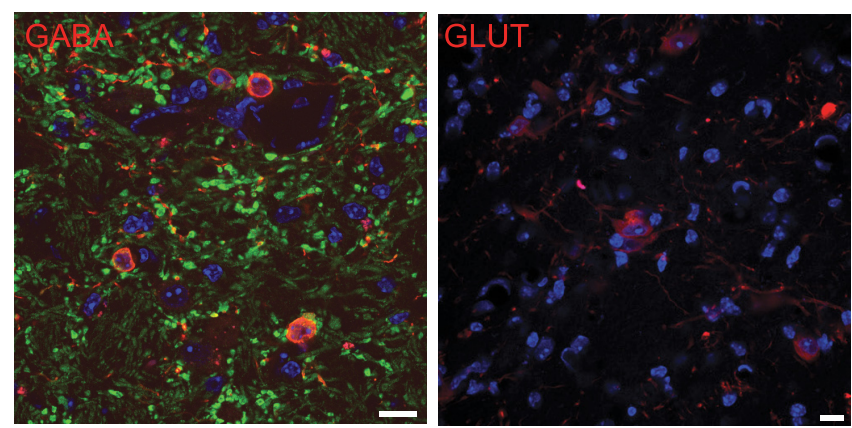

D

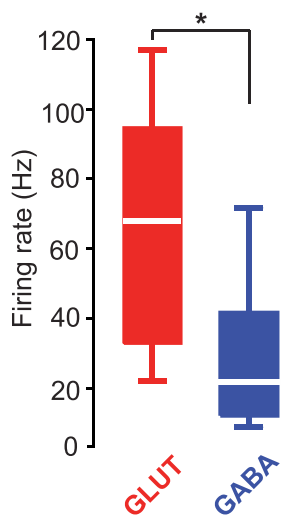

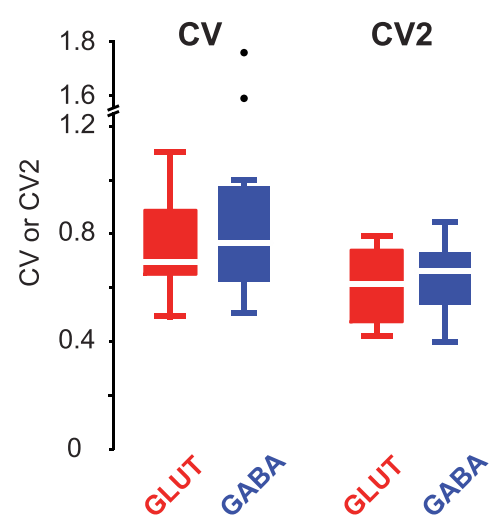

Figure 3. Juxtacellular recordings in transgenic mice expressing Chrimson in glutamatergic or GABAergic neurons identify the same two groups of CN neurons. $A$, Diagram of the experimental protocol for glass pipette recordings. GLUT and GABA neurons are identified by the activation of Chrimson in neurons recorded either in VGLUT2-cre or in GAD2-cre mice, respectively, after injection of an AAV9-hSyn-FLEX-ChrimsonR-tdTomato. Boxplots of the delay of the first spike from light onset (bottom right). B, GABA and GLUT neuron identification. Red represents Chrimson-tdTomato. Green represents Gad65/67*. Blue represents DAPI. Left, GABAergic neurons can be identified as smaller cells with both Chrimson-tdTomato and Gad65/67* expression. Right, Glutamatergic neurons are Chrimson-tdTomato. C, Mean of the averaged spike waveforms for GLUT and GABA neurons recorded using glass pipettes juxtacellular recordings. Note the similarity with waveforms recorded using tetrodes. $\boldsymbol{D}$, Boxplots of firing rates, $\mathrm{CV}$ and $\mathrm{CV} 2$, for the 2 groups: GLUT $(n=6)$ versus GABA $(n=11)$. Wilcoxon rank test firing rate: ${ }^{*} p=0.012 ; \mathrm{CV}: p=0.28 ; \mathrm{CV} 2: p=0.42$.

Fig. 2F and Fig. 3C; Table 1). Also, we confirmed that GLUT cells had a higher firing rate, comparable with the Group 1 neurons (mean: $66.6 \pm 39.7 \mathrm{~Hz} ; n=6$ ) than GABA cells (mean: $29.1 \pm 23$ $\mathrm{Hz} ; n=11$; Fig. $3 D ; p=0.012$, Wilcoxon rank test), which corresponded to the Group 2 neurons. No difference was observed in spike train regularity (Fig. $3 D$; mean $C V=0.76 \pm 0.22$ vs $0.90 \pm 0.42$; Wilcoxon rank test, $p=0.28$; mean CV2, $0.61 \pm$ 0.16 vs $0.64 \pm 0.15$; Wilcoxon rank test, $p=0.42$ ). These results demonstrate that GLUT and GABA neurons can be reliably discriminated solely by their spike waveform in extracellular recordings in awake animals. For these reasons, we will refer to the Group 1 neurons recorded in anesthetized L7-ChR2(H134R)YFP mice using tetrodes as GLUT-like neurons, and Group 2 neurons as GABA-like neurons.
Impact of synchronous PC firing on firing rate of GLUT-like and GABA-like neurons

In the L7-ChR2(H134R)-YFP mice, we combined tetrode recordings with a set of $\mathrm{PC}$ illumination protocols: periodic pulse trains at 40,65 , and $115 \mathrm{~Hz}$ (Fig. 4A). Most CN neurons (37 of 39) were inhibited by synchronous activation of PCs (Fig. 4B). The amount of inhibition did not depend on the baseline firing rate of the CN cells (GLUT-like: $r=-0.18, n=22, p=0.40$; GABAlike: $r=-0.06, n=17, p=0.83)$. When the stimulation frequency was increased (trains at 40,65, and $115 \mathrm{~Hz}$ ), the mean firing rate of $\mathrm{CN}$ neurons monotonically decreased for both GLUT-like and GABA-like cells (Fig. 4B). These results suggest that, while individual PCs do not strictly follow optogenetic stimulation at high frequency $(115 \mathrm{~Hz}$, see above), PC population 
A
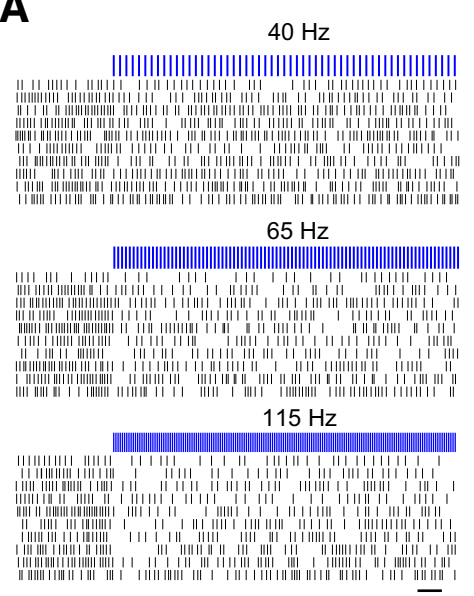

$10 \overline{\mathrm{ms}}$
B

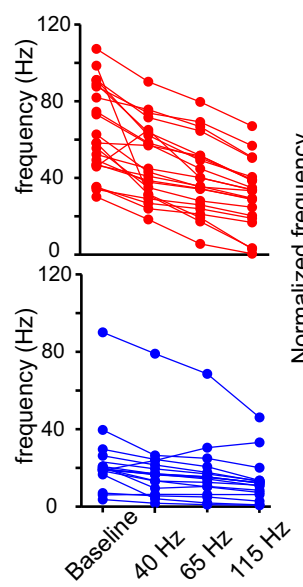

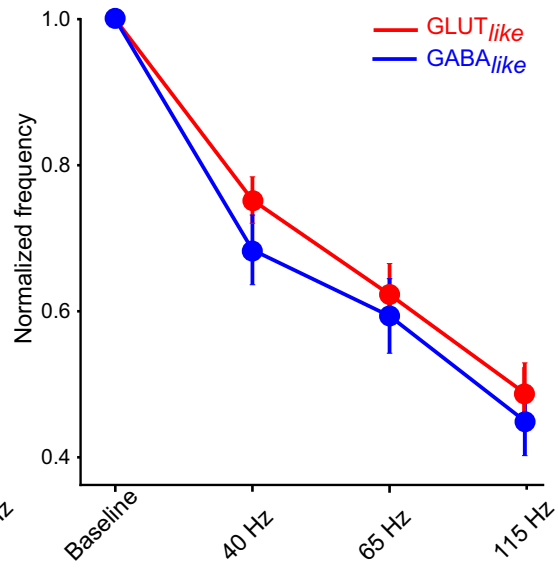

Figure 4. GLUT-like and GABA-like neuron firing rates are similarly affected by Purkinje cells. $A$, Raster plots of one example GLUT-like cell inhibited by trains ( $5 \mathrm{~ms}$ pulse length) of PCillumination at 40, 65, and $115 \mathrm{~Hz}$. B, GLUT-like and GABA-like are gradually inhibited by PC illumination. Left, Mean firing rate of individual units during illumination. Right, Averaged normalized firing rate of GLUT-like and GABA-like neuron population. Error bars indicate mean \pm SEM.

controls similarly GLUT-like and GABA-like neuron firing rates at all tested frequencies.

\section{GLUT-like neurons are synchronized and entrained by synchronous PC inputs}

We observed that some $\mathrm{CN}$ cells discharges could be time-locked to PC inputs during trains of stimulation, whereas others fired randomly during stimulation, albeit at lower rates (see two example cells in Fig. $5 A-D$ vs Fig. $5 E-H$ ). Indeed, ISI histograms estimated during illumination displayed a peak at the illumination frequency (Fig. 5C). We then computed spike time-locking by building the histogram of the spike times between 2 illuminations during trains of stimulation pulses (Fig. $5 B, F$ ). The Rayleigh test for histogram uniformity demonstrated that spiking in GLUTlike neurons was time-locked (i.e., synchronized) to PC illuminations $(p<0.01$ indicating nonuniform distribution for 22 of 22 cells at $40 \mathrm{~Hz}, 19$ of 22 cells at $65 \mathrm{~Hz}$, and 17 of 22 cells at 115 $\mathrm{Hz}$ ). By contrast, most GABA-like neurons spiked with uniform probability all through the interstimulus interval, indicating that these neurons were not synchronized to $\mathrm{PC}$ spiking input $>40 \mathrm{~Hz}$ ( 13 of 17 at $40 \mathrm{~Hz}, 10$ of 17 at $65 \mathrm{~Hz}, 14$ of 17 at $115 \mathrm{~Hz}$ ). To further quantify the spiking activity during trains of illuminations, we calculated the power spectra of the autocorrelogram (restricted to the time of illumination) for each cell (Fig. 5D, H). The spectra of spiking activity of $\mathrm{CN}$ neurons revealed that the GLUT-like neurons output followed the dominant input frequency (Fig. $5 D, I$ ) in that they were entrained by synchronous PC activity. By contrast, the spectra of GABA-like neurons was independent of the input frequencies (Fig. $5 H$ ), indicating that GABA-like neurons were not entrained by the PC neurons, at least not at the three different frequencies used in our experiments (compare Fig. 5D,H). Consistent with this, the power spectral density values at the stimulation frequencies were significantly larger for GLUT-like neurons than for GABA-like neurons (Fig. $5 I ; 40,65$, and $115 \mathrm{~Hz} ; p=2.6 \times 10^{-6}, p=3.7 \times 10^{-5}$ and $p=0.00076$, Wilcoxon rank test).

Because fixed-frequency stimulations are physiologically unlikely, we also illuminated the PCs in an irregular manner (illumination times drawn according to a homogeneous Poisson process with average rate of $40 \mathrm{~Hz}$ ). Because of the irregular illumination, the instantaneous rate of PCs discharges varied from 5 to $210 \mathrm{~Hz}$ (Fig. 6A). The distributions of IFRs for two examples of GLUT-like and GABA-like neurons during illumination and baseline are shown in Figure $6 B$. To test whether $\mathrm{CN}$ neurons were also entrained by irregular activity of PCs, we compared (using Kolmogorov-Smirnov test) the distributions of IFR of CN neurons during stimulation and the distribution of instantaneous illumination rate of PCs. If the CN cells would be entrained by PCs, then we should expect that the two distributions will be statistically similar. In 14 of 22 GLUT-like neurons, no differences $(p>0.05)$ were observed between the IFR histogram during illumination and the histogram of instantaneous stimulation frequencies, whereas in the 8 remaining cells, $p$ was $<0.05$ (Fig. $6 B, C)$. This indicates that a majority of GLUT-like cells $(63 \%)$ followed the PC discharges during stimulation (Fig. 6C). Conversely, in 15 of 17 GABA-like neurons, the IFR histogram during illumination was significantly different from the histogram of instantaneous stimulation frequencies $(p<0.05$; for 2 GABAlike neurons $p>0.05$; Fig. $6 C$ ), showing that most of these neurons did not follow the temporal PC discharge pattern during stimulation. Together, our findings demonstrate that most of the GLUT-like neurons were able to follow PC discharge in a temporally precise manner (fixed frequencies: $100 \%$ at $40 \mathrm{~Hz}, 86 \%$ at 65 $\mathrm{Hz}, 77 \%$ at $115 \mathrm{~Hz}$; $63 \%$ during irregular stimulation).

\section{Discussion}

Using in vivo extracellular recordings of $\mathrm{CN}$ neuronal activity in anesthetized and awake mice, we showed that putative GLUT and GABA neurons can be discriminated using simple features of extracellular spike waveforms and respond differently to PC inputs.

\section{Evidence for the discrimination of GLUT versus GABA neurons in $\mathrm{CN}$}

In our first series of experiments, using tetrodes in anesthetized mice and without prior information on cell type identity, we adopted a conservative approach, leading to a dataset of 39 identified CN cells, with only few multiple cells recorded on a single tetrode. This difficulty is likely the result of the spatial organization of the electrodes combined with the facts that $\mathrm{CN}$ neurons are densely packed. Silicon probes have flat shanks that do not allow a $3 \mathrm{D}$ positioning of the recording sites as opposed to, for 
A

C

GLUT $_{\text {like }}$

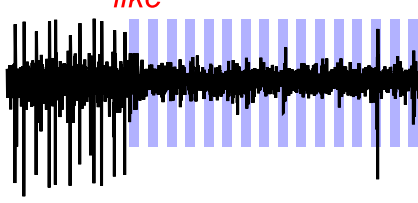

B

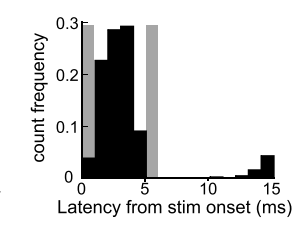

ISI Distribution
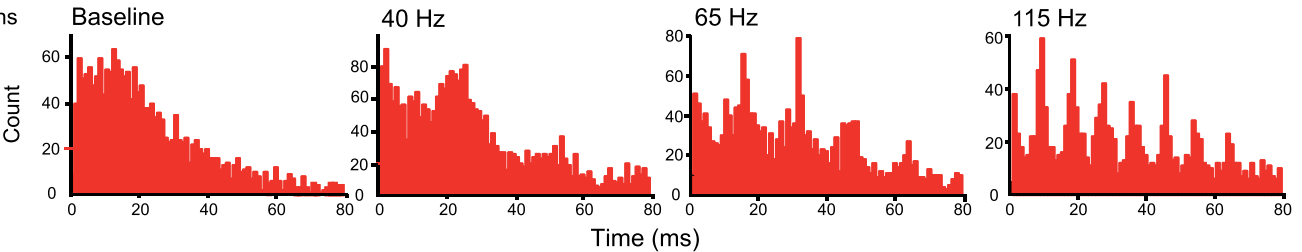

D Power Spectra
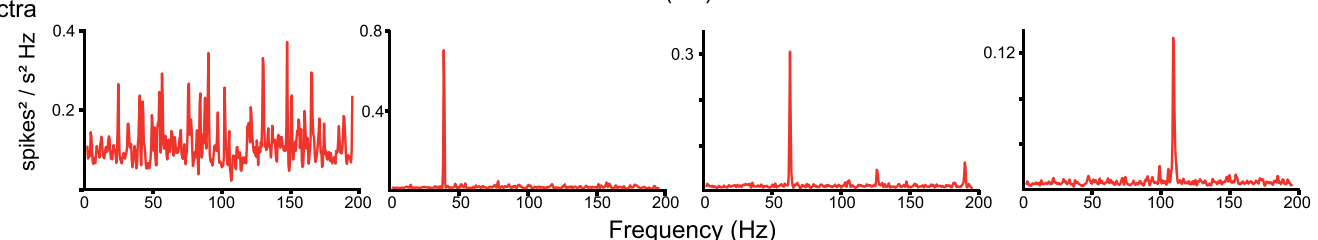

E

GABA $_{\text {ike }}$

F
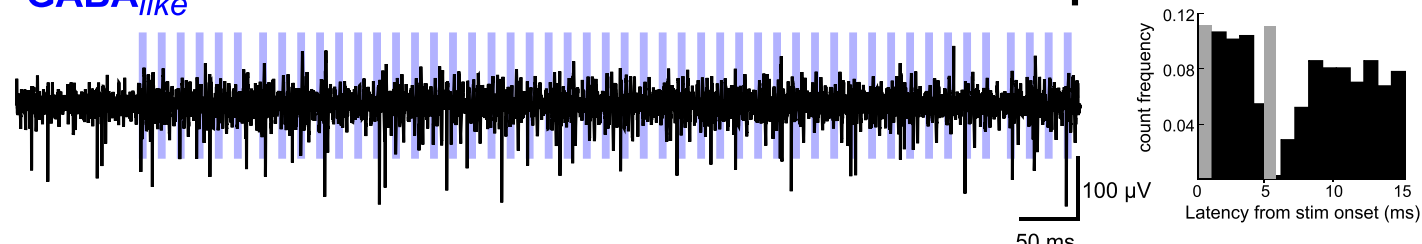

G

ISI Distributions Baseline

$40 \mathrm{~Hz}$

$65 \mathrm{~Hz}$
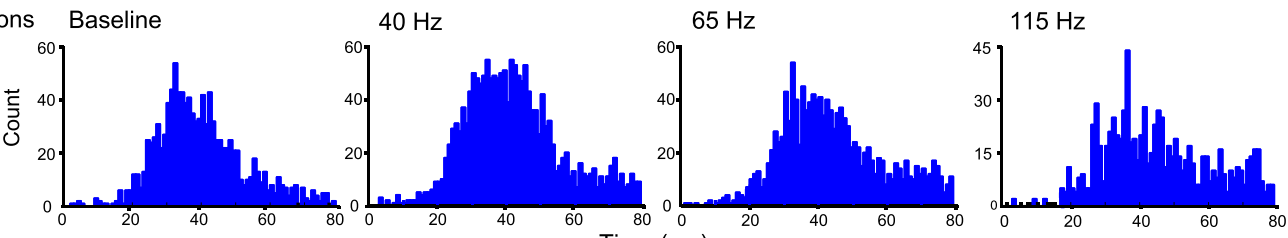

H Power Spectra
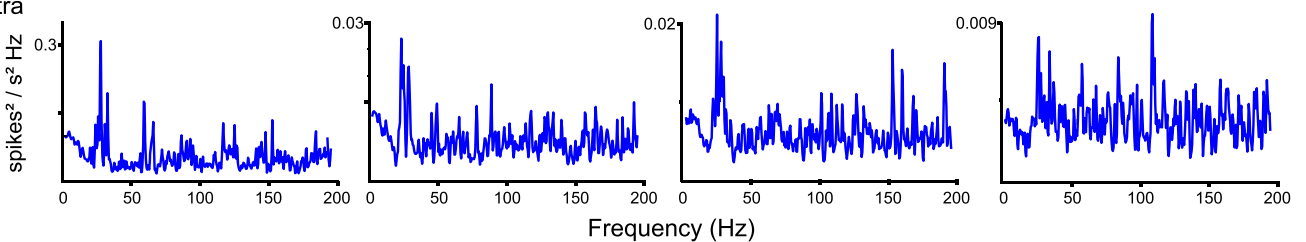

I

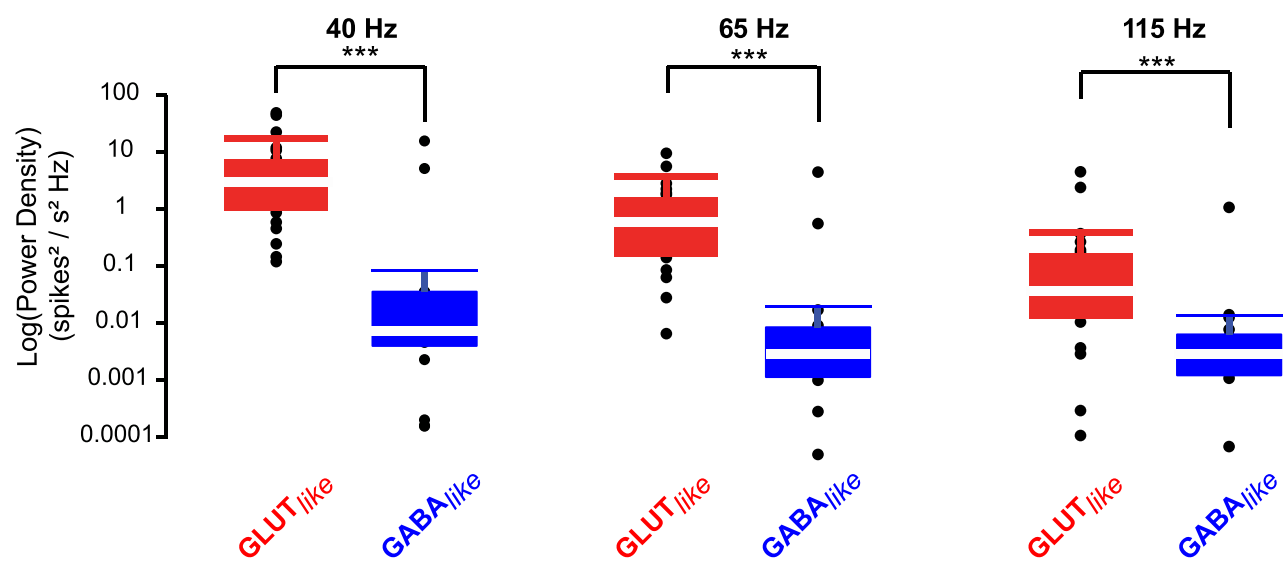

Figure 5. Differential temporal coding by GLUT-like and GABA-like neurons. Nuclear neuron discharges during trains of PC illumination at 40,65 , and $115 \mathrm{~Hz}$. A-D, Example of GLUT-like neuron. $\boldsymbol{A}$, Raw trace from a GLUT-like unit during illumination (blue bars) at $65 \mathrm{~Hz}$. $\boldsymbol{B}$, Histogram of the spike latency from stimulation onset. Gray bar represents the position of the subtracted stimulation artifact. C, ISI histograms at 40,65, and $115 \mathrm{~Hz}$. D, Power spectra of the unit autocorrelogram at the same frequencies. $\boldsymbol{E}$ - $\boldsymbol{H}$, Same analyses for a GABA-like neuron. $\boldsymbol{I}$, Boxplot of averaged power density for GLUT-like and GABA-like neurons at 40,65, and $115 \mathrm{~Hz}$. White line indicates median. Box limit indicates first (Q1) and third (Q3) quartiles. Whiskers represent 10th and 90 th percentiles. Wilcoxon rank tests: ${ }^{* * *} p=2.6 \times 10^{-6},{ }^{* * *} p=3.7 \times 10^{-5},{ }^{* * *} p=0.00076$. 
A

GLUT like neurons

|| |||| | | || |||| | ||| |||||||||||| | | ||| ||||||||||||||| |||||||| | ||||| ||| | | | |||

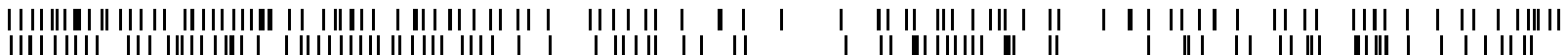

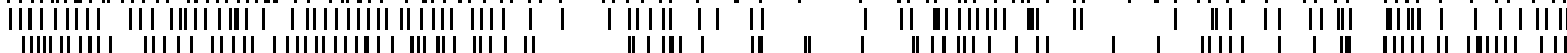
1 Tillil

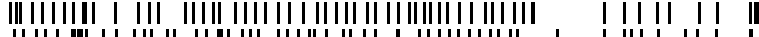
|I || | ||| || ||| || || || ||| |||||| || || | || |||||||||| | | ||| | || | | | |||||||||||||||| ||||||||||||||||| ||||||||||||||||||||||||||||||||||| | | ||||| | | | | | || ||||| | |||||||| || |||| |||||||| ||| |||||| ||||| ||| |||||||| ||||||||| ||||| || |||||||||||||||||| |||||||||||||| ||||||||||||| | ||| | |||||||||||||||||| | | | ||| |||||||||| ||||||| | ||||| | ||||| ||| || |||| |||||||| IIIIII I II II III II III III | |||||||||||| ||| || ||| |||| |||||||||||||||||||||||||| | ||| |||| ||||| ||

\section{$\mathrm{GABA}_{\text {like }}$ neurons}

I II II I I I I I

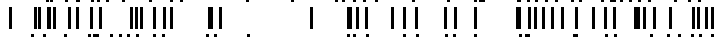
II I I|| IIII II II I I II II II I II I II I II III I I

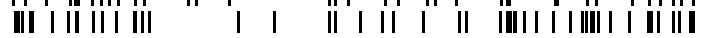

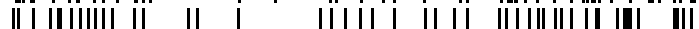
| || | |||||||||||| | ||| || || | ||| ||||||| | | ||| |||| ||| ||||

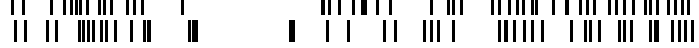

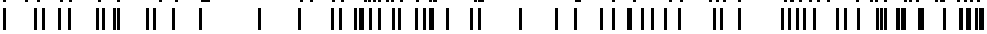

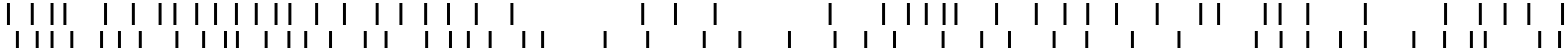

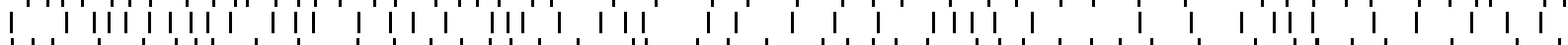

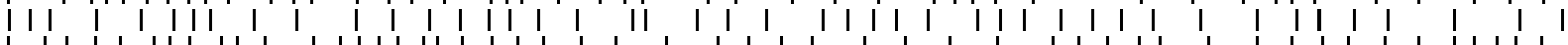

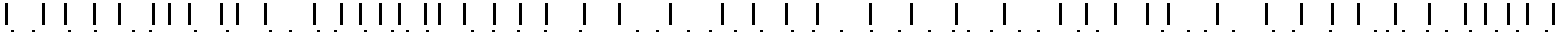

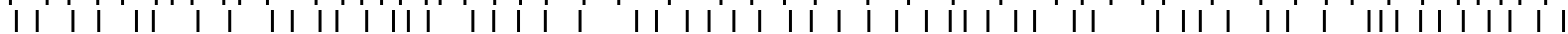

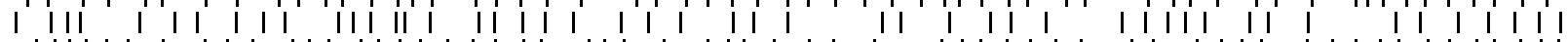

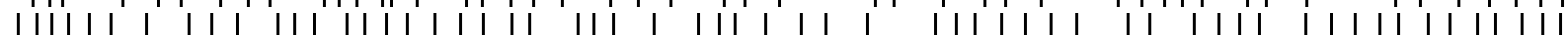

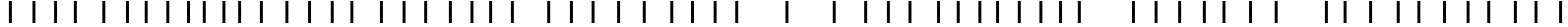

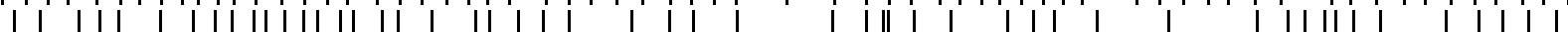

B

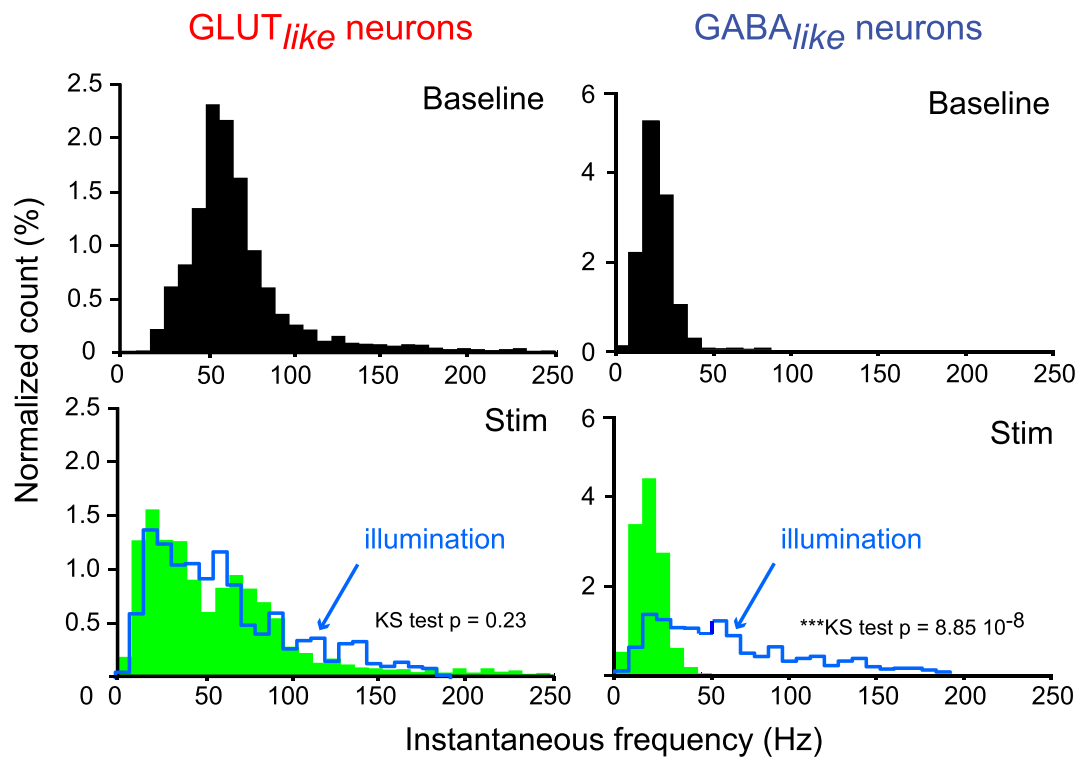

C $10 \overline{\mathrm{ms}}$

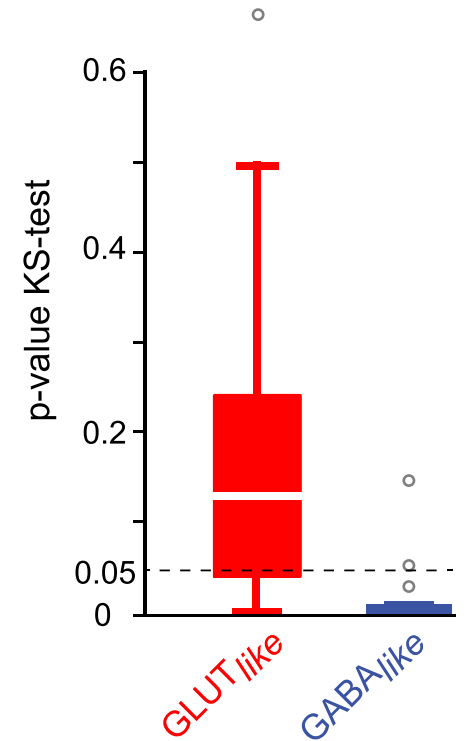

Figure 6. GLUT-like neurons can follow a wide range of frequencies. $A$, Example of raster plots for one GLUT-like neuron and one GABA-like neuron during train of irregular $P C$ illuminations ( $5 \mathrm{~ms}$ pulse length; illumination times drawn according to a homogeneous Poisson process with average rate of $40 \mathrm{~Hz}$ ). $\boldsymbol{B}$, Histogram of the normalized IFR for the GLUT-like neuron (left) and the GABA-like neuron (right) during baseline (top, black) and during the trains of $\mathrm{PC}$ illuminations (bottom, green) superimposed to the IFR histogram of the stimulation (blue cityscape). Note the overlap of the IFR histogram for the GLUT-like neuron with the IFR histogram of the stimulation. KS test $p, p$ value of the Kolmogorov-Smirnov test. C, Boxplot of the $p$ value of the Kolmogorov-Smirnov test between IFR histogram of cerebellar neurons (for GLUT-like and GABA-like neurons) during PC stimulation and IFR histogram of the stimulation.

example, quartz electrodes, which have a tetrahedral architecture (Gao et al., 2012), limiting the discriminating power of such tetrodes. Nevertheless, PCA followed by a hierarchical clustering analysis allowed us to identify two clusters of spike waveforms (Group 1: narrow action potential width; Group 2: broader action potential), forming two populations of neurons with different mean firing rates. In a second series of experiments in awake animals, we specifically expressed Chrimson in GLUT and GABA neurons; and using glass pipettes and tetrodes, we identified the same properties as in the aforementioned Group 1 and Group 2 cells: GLUT neurons had a narrow spike width and high firing rate, whereas GABA neurons had a broader spike width and a low firing rate as opposed to the neocortex in which pyramidal GLUT cells have broader spikes and GABA cells have narrow spikes (Barthó et al., 2004). These findings are in agreement with in vitro intracellular recordings (Uusisaari et al., 2007). In whole-cell current-clamp configuration, Uusisaari et al. (2007) found that non-GABAergic big cells (presumably the glutamatergic projection cells) had narrow action potential widths and higher firing rate compared with GABAergic cells, having larger action potential widths and lower firing rate. Although glycinergic projection cells have also narrow spikes and similar maximum firing rates as glutamatergic projection neurons (Bagnall et al., 2009), they were identified in the ventral part of the rostral medial nucleus, 
whereas our recordings were mainly located in caudal dorsal areas of the nucleus (Fig. $1 B$ ). However, given that smaller glutamatergic neurons generally fire at low frequency (Uusisaari et al., 2007) and that our PCA analysis was based on a limited dataset, we cannot rule out that some of the cells that were recorded using tetrodes may belong to other classes of $\mathrm{CN}$ neurons. Still, as independent analyses of two different datasets recorded in anesthetized and awake animals identified similar groups based on biophysical parameters (spike waveform and firing rate), it is the most parsimonious to postulate that most of Group 1 GLUT-like cells are glutamatergic projection neurons of the cerebellum. We then note that, in all recording conditions, including acute brain slices in vitro (Uusisaari and Knöpfel, 2011), anesthetized and awake animals, the same biophysical segregation (spike waveform and mean firing rate) between GABA and GLUT neurons has been observed. This indicates that these properties must be a hallmark of $\mathrm{CN}$ physiology as they were observed in different physiological conditions.

Nonglutamatergic cells can be split into GABAergic nucleoolivary projection neurons, local GABA/glycinergic, and nucleocortical glycynergic neurons (Uusisaari and Knöpfel, 2011). Glycinergic nucleocortical cells are probably mostly silent (Ankri et al., 2015) and nucleo-olivary neurons are most prominently distributed in the ventrolateral part of the cerebellar nuclei (Fredette and Mugnaini, 1991; De Zeeuw et al., 1997; Najac and Raman, 2015). Therefore, although we cannot rule out that some cells may belong to other classes of $\mathrm{CN}$ neurons, we postulate that most of the GABA-like cells belong to the local GABAergic group of neurons, and demonstrate that they are reliably inhibited by $\mathrm{PC}$ inputs in vivo.

\section{Different coding strategies in $\mathrm{CN}$ neurons of the medial nucleus}

$\mathrm{CN}$ neurons integrate excitatory inputs from mossy and climbing fibers, and inhibitory inputs from PCs and local interneurons. It was recently demonstrated that excitatory afferents are responsible for half of the action potentials in CN cells (YardenRabinowitz and Yarom, 2017); the other half is intrinsically elicited since CN neurons are spontaneously active (Thach, 1968; Aizenman and Linden, 1999; Raman et al., 2000; Alviña et al., 2009; Sangrey and Jaeger, 2010; Tadayonnejad et al., 2010; Zheng and Raman, 2010; Boehme et al., 2011; Engbers et al., 2013). Although the existence of a PC inhibitory control of CN cells is generally accepted (Ito et al., 1970; Ito, 1984; Gauck and Jaeger, 2000; Czubayko et al., 2001; Telgkamp and Raman, 2002; Pedroarena and Schwarz, 2003; Rowland and Jaeger, 2005; Blenkinsop and Lang, 2011; Person and Raman, 2011; Chaumont et al., 2013), the question of whether this inhibition gradually decreases $\mathrm{CN}$ neurons mean firing rate (rate code), delimits a temporal window for neuron discharge (temporal code), or both is still debated (De Zeeuw et al., 2008, 2011). CN neurons can follow ongoing movement kinematics (Thach, 1968, 1970a; Armstrong and Edgley, 1984; Rowland and Jaeger, 2005) and can be entrained by synchronous PCs, as suggested by recordings showing correlated firing of PC and CN neurons (Thach, 1970a,b; Armstrong and Edgley, 1984; McDevitt et al., 1987). Recently, several studies also demonstrated that rate and time coding strategies can be multiplexed (Sudhakar et al., 2015; Abbasi et al., 2017; Brown and Raman, 2018). Our study is in agreement with previous experiments showing that large $\mathrm{CN}$ neurons (presumably glutamatergic projection cells) are entrained by synchronous PCs, whereas at high frequencies GABAergic neurons are essentially inhibited (Person and Raman, 2011; Najac and Raman, 2015;
Sarnaik and Raman, 2018). However, in some in vivo recordings, it was shown that synchronized PCs entrain large CN neurons without affecting their overall firing rates (Person and Raman, 2011; Sarnaik and Raman, 2018) unless climbing fiber inputs were involved (Hoebeek et al., 2010; Bengtsson et al., 2011; Tang et al., 2016, 2019). This absence of an effect on CN neuron firing rates might be accounted for by the slow kinetics of the ChR2 currents in PCs that would fail to elicit a spike at each illumination at high frequency or by a reduced number of activated PCs. Notably, in an vitro study, an inhibitory effect of increasing PC firing rate with constant synchrony has been observed (Wu and Raman, 2017). In our study, while the mean firing rates of individual PCs do not follow illumination at high frequency (i.e., mean firing rate $=57 \mathrm{~Hz}$ at $115 \mathrm{~Hz}$ ), PCs are entrained at the illumination frequency up to $115 \mathrm{~Hz}$ at the population level (Fig. 1), and GLUT-like cells are both inhibited and entrained by blue light activation. These results suggest that PCs can fire at $115 \mathrm{~Hz}$ but skip some cycles as during high-frequency oscillations (de Solages et al., 2008) and that converging inputs from 30 to 50 PCs to a given CN neuron (Person and Raman, 2011) ensure its entrainment at $115 \mathrm{~Hz}$. However, the lack of a strict PC entrainment at $115 \mathrm{~Hz}$ likely led to a loose synchronization of PC firing that may promote the concomitant inhibition of $\mathrm{CN}$ neurons, as observed by Person and Raman (2011) (see stimulation at $100 \mathrm{~Hz}$ in their Fig. 2D). Finally, the apparent difference in PC influence on $\mathrm{CN}$ neurons firing rate with some in vivo recordings (Person and Raman, 2011; Sarnaik and Raman, 2018) might originate in the different targeted nucleus, the medial instead of the interposed nucleus. In the medial nucleus, PC axons spread out in a wide area of the nucleus (Sugihara et al., 2009), as opposed to PC projections in the interposed nucleus (Sarnaik and Raman, 2018). This configuration likely leads to a few millisecond (2-3 $\mathrm{ms}$ ) jitter in action potential conduction time on PC axons, which is in line with the loose synchronization mediated by ChR2 activation in PCs (Chaumont et al., 2013). These jitters may slightly desynchronize PC inputs and result in a mild inhibition of $\mathrm{CN}$ neurons (De Zeeuw et al., 2008), as shown by Person and Raman (2011). Although the light-induced jittering in PC inputs is not physiological, it may mimic the physiological jitter in PC synchrony when PCs are excited by clusters of activated granule cells (Heck et al., 2007; Valera et al., 2016). Since GLUT-like cells are both gradually inhibited and entrained by a population of PC inputs discharging together (de Solages et al., 2008), we conclude that they multiplex a population rate and temporal code strategies. Conversely, local GABA-like cells are inhibited by PC inputs, but not entrained at high frequencies, in agreement with in vitro recordings of GABAergic nucleo-olivary cells (Najac and Raman, 2015). As PC frequency increases, local GABA interneurons might be fully inhibited, releasing the local inhibitory brake on projection GLUT cells that are entrained by PC inputs. These differential coding strategies between $\mathrm{CN}$ neurons might favor temporal precision of cerebellar outputs associated with specific features of movement control in the medial nucleus.

\section{References}

Abbasi S, Hudson AE, Maran SK, Cao Y, Abbasi A, Heck DH, Jaeger D (2017) Robust transmission of rate coding in the inhibitory Purkinje cell to cerebellar nuclei pathway in awake mice. PLoS Comput Biol 13:e1005578.

Aizenman CD, Linden DJ (1999) Regulation of the rebound depolarization and spontaneous firing patterns of deep nuclear neurons in slices of rat cerebellum. J Neurophysiol 82:1697-1709.

Allen GI, Tsukahara N (1974) Cerebrocerebellar communication systems. Physiol Rev 54:957-1006.

Alviña K, Walter JT, Kohn A, Ellis-Davies G, Khodakhah K (2008) Ques- 
tioning the role of rebound firing in the cerebellum. Nat Neurosci 11:1256-1258.

Alviña K, Ellis-Davies G, Khodakhah K (2009) T-type calcium channels mediate rebound firing in intact deep cerebellar neurons. Neuroscience 158:635-641.

Ankri L, Husson Z, Pietrajtis K, Proville R, Léna C, Yarom Y, Dieudonné S, Uusisaari MY (2015) A novel inhibitory nucleo-cortical circuit controls cerebellar Golgi cell activity. Elife 4:e06262.

Armstrong DM, Edgley SA (1984) Discharges of nucleus interpositus neurones during locomotion in the cat. J Physiol 351:411-432.

Bagnall MW, Zingg B, Sakatos A, Moghadam SH, Zeilhofer HU, du Lac S (2009) Glycinergic projection neurons of the cerebellum. J Neurosci 29:10104-10110.

Barthó P, Hirase H, Monconduit L, Zugaro M, Harris KD, Buzsáki G (2004) Characterization of neocortical principal cells and interneurons by network interactions and extracellular features. J Neurophysiol 92:600-608.

Bastian AJ (2006) Learning to predict the future: the cerebellum adapts feedforward movement control. Curr Opin Neurobiol 16:645-649.

Bengtsson F, Jörntell H (2014) Specific relationship between excitatory inputs and climbing fiber receptive fields in deep cerebellar nuclear neurons. PLoS One 9:e84616.

Bengtsson F, Ekerot CF, Jörntell H (2011) In vivo analysis of inhibitory synaptic inputs and rebounds in deep cerebellar nuclear neurons. PLoS One 6:e18822.

Blenkinsop TA, Lang EJ (2011) Synaptic action of the olivocerebellar system on cerebellar nuclear spike activity. J Neurosci 31:14708-14720.

Boehme R, Uebele VN, Renger JJ, Pedroarena C (2011) Rebound excitation triggered by synaptic inhibition in cerebellar nuclear neurons is suppressed by selective T-type calcium channel block. J Neurophysiol 106: 2653-2661.

Brooks VB, Thach WT (2011) Cerebellar control of posture and movement. In: Comprehensive physiology, pp 877-946. Hoboken, NJ: Wiley.

Brown ST, Raman IM (2018) Sensorimotor integration and amplification of reflexive whisking by well-timed spiking in the cerebellar corticonuclear circuit. Neuron 99:564-575.e2.

Canto CB, Witter L, De Zeeuw CI (2016) Whole-cell properties of cerebellar nuclei neurons in vivo. PLoS One 11:e0165887.

Chaumont J, Guyon N, Valera AM, Dugué GP, Popa D, Marcaggi P, Gautheron V, Reibel-Foisset S, Dieudonné S, Stephan A, Barrot M, Cassel JC, Dupont JL, Doussau F, Poulain B, Selimi F, Léna C, Isope P (2013) Clusters of cerebellar Purkinje cells control their afferent climbing fiber discharge. Proc Natl Acad Sci U S A 110:16223-16228.

Chen X, Kovalchuk Y, Adelsberger H, Henning HA, Sausbier M, Wietzorrek G, Ruth P, Yarom Y, Konnerth A (2010) Disruption of the olivocerebellar circuit by Purkinje neuron-specific ablation of BK channels. Proc Natl Acad Sci U S A 107:12323-12328.

Czubayko U, Sultan F, Thier P, Schwarz C (2001) Two types of neurons in the rat cerebellar nuclei as distinguished by membrane potentials and intracellular fillings. J Neurophysiol 85:2017-2029.

de Solages C, Szapiro G, Brunel N, Hakim V, Isope P, Buisseret P, Rousseau C, Barbour B, Léna C (2008) High-frequency organization and synchrony of activity in the Purkinje cell layer of the cerebellum. Neuron 58:775-788.

De Zeeuw CI, Van Alphen AM, Hawkins RK, Ruigrok TJ (1997) Climbing fibre collaterals contact neurons in the cerebellar nuclei that provide a GABAergic feedback to the inferior olive. Neuroscience 80:981-986.

De Zeeuw CI, Hoebeek FE, Schonewille M (2008) Causes and consequences of oscillations in the cerebellar cortex. Neuron 58:655-658.

De Zeeuw CI, Hoebeek FE, Bosman LW, Schonewille M, Witter L, Koekkoek SK (2011) Spatiotemporal firing patterns in the cerebellum. Nat Rev Neurosci 12:327-344.

Engbers JD, Anderson D, Zamponi GW, Turner RW (2013) Signal processing by T-type calcium channel interactions in the cerebellum. Front Cell Neurosci 7:230.

Fredette BJ, Mugnaini E (1991) The GABAergic cerebello-olivary projection in the rat. Anat Embryol (Berl) 184:225-243.

Gao H, de Solages C, Lena C (2012) Tetrode recordings in the cerebellar cortex. J Physiol Paris 106:128-136.

Garcia S, Fourcaud-Trocmé N (2009) OpenElectrophy: an electrophysiological data- and analysis-sharing framework. Front Neuroinform 3:14.

Gauck V, Jaeger D (2000) The control of rate and timing of spikes in the deep cerebellar nuclei by inhibition. J Neurosci 20:3006-3016.
Glickstein M, Sultan F, Voogd J (2011) Functional localization in the cerebellum. Cortex 47:59-80.

Heck DH, Thach WT, Keating JG (2007) On-beam synchrony in the cerebellum as the mechanism for the timing and coordination of movement. Proc Natl Acad Sci U S A 104:7658-7663.

Hoebeek FE, Stahl JS, van Alphen AM, Schonewille M, Luo C, Rutteman M, van den Maagdenberg AM, Molenaar PC, Goossens HH, Frens MA, De Zeeuw CI (2005) Increased noise level of Purkinje cell activities minimizes impact of their modulation during sensorimotor control. Neuron 45:953-965.

Hoebeek FE, Witter L, Ruigrok TJ, De Zeeuw CI (2010) Differential olivocerebellar cortical control of rebound activity in the cerebellar nuclei. Proc Natl Acad Sci U S A 107:8410-8415.

Hong S, Negrello M, Junker M, Smilgin A, Thier P, De Schutter E (2016) Multiplexed coding by cerebellar Purkinje neurons. Elife 5:e13810.

Husson Z, Rousseau CV, Broll I, Zeilhofer HU, Dieudonné S (2014) Differential GABAergic and glycinergic inputs of inhibitory interneurons and Purkinje cells to principal cells of the cerebellar nuclei. J Neurosci 34:9418-9431.

Ito M (1984) The cerebellum and neural control. New York: Raven.

Ito M, Yoshida M, Obata K, Kawai N, Udo M (1970) Inhibitory control of intracerebellar nuclei by the Purkinje cell axons. Exp Brain Res 10:64-80.

Kelly RM, Strick PL (2003) Cerebellar loops with motor cortex and prefrontal cortex of a nonhuman primate. J Neurosci 23:8432-8444.

Klapoetke NC, Murata Y, Kim SS, Pulver SR, Birdsey-Benson A, Cho YK, Morimoto TK, Chuong AS, Carpenter EJ, Tian Z, Wang J, Xie Y, Yan Z, Zhang Y, Chow BY, Surek B, Melkonian M, Jayaraman V, ConstantinePaton M, Wong GK, et al. (2014) Independent optical excitation of distinct neural populations. Nat Methods 11:338-346.

Lê S, Josse J, Husson F (2008) FactoMineR: an R package for multivariate analysis. J Stat Softw 25:1-18.

Mardinly AR, Oldenburg IA, Pégard NC, Sridharan S, Lyall EH, Chesnov K, Brohawn SG, Waller L, Adesnik H (2018) Precise multimodal optical control of neural ensemble activity. Nat Neurosci 21:881-893.

McCormick DA, Thompson RF (1984) Cerebellum: essential involvement in the classically conditioned eyelid response. Science 223:296-299.

McDevitt CJ, Ebner TJ, Bloedel JR (1987) Changes in the responses of cerebellar nuclear neurons associated with the climbing fiber response of Purkinje cells. Brain Res 425:14-24.

Millman KJ, Aivazis M (2011) Python for scientists and engineers. Comput Sci Eng 13:9-12.

Najac M, Raman IM (2015) Integration of Purkinje cell inhibition by cerebellar nucleo-olivary neurons. J Neurosci 35:544-549.

Pedregosa F, Varoquaux G, Gramfort A, Michel V, Thirion B, Grisel O, Blondel M, Prettenhofer P, Weiss R, Dubourg V, Vanderplas J, Passos A, Cournapeau D, Brucher M, Perrot M, Duchesnay É (2011) Scikit-learn: machine learning in python. J Mach Learn Res 12:2825-2830.

Pedroarena CM, Schwarz C (2003) Efficacy and short-term plasticity at GABAergic synapses between Purkinje and cerebellar nuclei neurons. J Neurophysiol 89:704-715.

Person AL, Raman IM (2011) Purkinje neuron synchrony elicits timelocked spiking in the cerebellar nuclei. Nature 481:502-505.

Person AL, Raman IM (2012) Synchrony and neural coding in cerebellar circuits. Front Neural Circuits 6:97.

Proville RD, Spolidoro M, Guyon N, Dugué GP, Selimi F, Isope P, Popa D, Léna C (2014) Cerebellum involvement in cortical sensorimotor circuits for the control of voluntary movements. Nat Neurosci 17:12331239.

R Core Team (2015) R: the R Project for Statistical Computing. Available at https://www.r-project.org/.

Raman IM, Gustafson AE, Padgett D (2000) Ionic currents and spontaneous firing in neurons isolated from the cerebellar nuclei. J Neurosci 20: 9004-9016.

Rasmussen A, Zucca R, Johansson F, Jirenhed DA, Hesslow G (2015) Purkinje cell activity during classical conditioning with different conditional stimuli explains central tenet of Rescorla-Wagner model [corrected]. Proc Natl Acad Sci U S A 112:14060-14065.

Rowland NC, Jaeger D (2005) Coding of tactile response properties in the rat deep cerebellar nuclei. J Neurophysiol 94:1236-1251.

Rowland NC, Jaeger D (2008) Responses to tactile stimulation in deep cerebellar nucleus neurons result from recurrent activation in multiple pathways. J Neurophysiol 99:704-717. 
Sangrey T, Jaeger D (2010) Analysis of distinct short and prolonged components in rebound spiking of deep cerebellar nucleus neurons. Eur J Neurosci 32:1646-1657.

Sarnaik R, Raman IM (2018) Control of voluntary and optogenetically perturbed locomotion by spike rate and timing of neurons of the mouse cerebellar nuclei. Elife 7:e29546.

Schmahmann JD, Pandya DN (1997) The cerebrocerebellar system. Int Rev Neurobiol 41:31-60.

Shin SL, De Schutter E (2006) Dynamic synchronization of Purkinje cell simple spikes. J Neurophysiol 96:3485-3491.

Spencer RM, Zelaznik HN, Diedrichsen J, Ivry RB (2003) Disrupted timing of discontinuous but not continuous movements by cerebellar lesions. Science 300:1437-1439.

Steuber V, Jaeger D (2013) Modeling the generation of output by the cerebellar nuclei. Neural Netw 47:112-119.

Steuber V, Mittmann W, Hoebeek FE, Silver RA, De Zeeuw CI, Häusser M, De Schutter E (2007) Cerebellar LTD and pattern recognition by Purkinje cells. Neuron 54:121-136.

Sudhakar SK, Torben-Nielsen B, De Schutter E (2015) Cerebellar nuclear neurons use time and rate coding to transmit Purkinje neuron pauses. PLoS Comput Biol 11:e1004641.

Sugihara I, Fujita H, Na J, Quy PN, Li BY, Ikeda D (2009) Projection of reconstructed single Purkinje cell axons in relation to the cortical and nuclear aldolase $\mathrm{C}$ compartments of the rat cerebellum. J Comp Neurol 512:282-304.

Tadayonnejad R, Anderson D, Molineux ML, Mehaffey WH, Jayasuriya K, Turner RW (2010) Rebound discharge in deep cerebellar nuclear neurons in vitro. Cerebellum 9:352-374.

Tang T, Suh CY, Blenkinsop TA, Lang EJ (2016) Synchrony is key: complex spike inhibition of the deep cerebellar nuclei. Cerebellum 15:10-13.

Tang T, Blenkinsop TA, Lang EJ (2019) Complex spike synchrony dependent modulation of rat deep cerebellar nuclear activity. Elife 8:e40101.

Telgkamp P, Raman IM (2002) Depression of inhibitory synaptic transmission between Purkinje cells and neurons of the cerebellar nuclei. J Neurosci 22:8447-8457.

Ten Brinke MM, Boele HJ, Spanke JK, Potters JW, Kornysheva K, Wulff P, IJpelaar AC, Koekkoek SK, De Zeeuw CI (2015) Evolving models of Pavlovian conditioning: cerebellar cortical dynamics in awake behaving mice. Cell Rep 13:1977-1988.

Ten Brinke MM, Heiney SA, Wang X, Proietti-Onori M, Boele HJ, Bakermans J, Medina JF, Gao Z, De Zeeuw CI (2017) Dynamic modulation of activity in cerebellar nuclei neurons during Pavlovian eyeblink conditioning in mice. Elife 6:e28132.
Thach WT (1968) Discharge of Purkinje and cerebellar nuclear neurons during rapidly alternating arm movements in the monkey. J Neurophysiol 31:785-797.

Thach WT (1970a) Discharge of cerebellar neurons related to two maintained postures and two prompt movements: I. Nuclear cell output. J Neurophysiol 33:527-536.

Thach WT (1970b) Discharge of cerebellar neurons related to two maintained postures and two prompt movements: II. Purkinje cell output and input. J Neurophysiol 33:537-547.

Thach WT, Goodkin HP, Keating JG (1992) The cerebellum and the adaptive coordination of movement. Annu Rev Neurosci 15:403-442.

Uusisaari M, Knöpfel T (2008) GABAergic synaptic communication in the GABAergic and non-GABAergic cells in the deep cerebellar nuclei. Neuroscience 156:537-549.

Uusisaari M, Knöpfel T (2011) Functional classification of neurons in the mouse lateral cerebellar nuclei. Cerebellum 10:637-646.

Uusisaari M, Obata K, Knöpfel T (2007) Morphological and electrophysiological properties of GABAergic and non-GABAergic cells in the deep cerebellar nuclei. J Neurophysiol 97:901-911.

Valera AM, Binda F, Pawlowski SA, Dupont JL, Casella JF, Rothstein JD, Poulain B, Isope P (2016) Stereotyped spatial patterns of functional synaptic connectivity in the cerebellar cortex. Elife 5:e09862.

Walter JT, Khodakhah K (2006) The linear computational algorithm of cerebellar Purkinje cells. J Neurosci 26:12861-12872.

Walter JT, Alviña K, Womack MD, Chevez C, Khodakhah K (2006) Decreases in the precision of Purkinje cell pacemaking cause cerebellar dysfunction and ataxia. Nat Neurosci 9:389-397.

White JJ, Sillitoe RV (2017) Genetic silencing of olivocerebellar synapses causes dystonia-like behaviour in mice. Nat Commun 8:14912.

White JJ, Arancillo M, Stay TL, George-Jones NA, Levy SL, Heck DH, Sillitoe RV (2014) Cerebellar zonal patterning relies on Purkinje cell neurotransmission. J Neurosci 34:8231-8245.

Wu Y, Raman IM (2017) Facilitation of mossy fibre-driven spiking in the cerebellar nuclei by the synchrony of inhibition. J Physiol 595:5245-5264.

Yarden-Rabinowitz Y, Yarom Y (2017) In vivo analysis of synaptic activity in cerebellar nuclei neurons unravels the efficacy of excitatory inputs. J Physiol 595:5945-5963.

Yeo CH, Lobo DH, Baum A (1997) Acquisition of a new-latency conditioned nictitating membrane response-major, but not complete, dependence on the ipsilateral cerebellum. Learn Mem 3:557-577.

Zheng N, Raman IM (2010) Synaptic inhibition, excitation, and plasticity in neurons of the cerebellar nuclei. Cerebellum 9:56-66. 